\title{
A General Theory of Non-equilibrium Dynamics of Lipid-protein Fluid Membranes
}

\author{
Michael A. Lomholt ${ }^{\mathrm{a}}$, Per L. Hansen ${ }^{\mathrm{b}}$, and Ling Miao ${ }^{\mathrm{c}}$ \\ The MEMPHYS Center for Biomembrane Physics, Physics Department, University of Southern Denmark, \\ DK-5230 Odense M, Denmark
}

\begin{abstract}
We present a general and systematic theory of non-equilibrium dynamics of multi-component fluid membranes, in general, and membranes containing transmembrane proteins, in particular. Developed based on a minimal number of principles of statistical physics and designed to be a meso/macroscopicscale effective description, the theory is formulated in terms of a set of equations of hydrodynamics and linear constitutive relations. As a particular emphasis of the theory, the equations and the constitutive relations address both the thermodynamic and the hydrodynamic consequences of the unconventional material characteristics of lipid-protein membranes and contain proposals as well as predictions which have not yet been made in already existed work on membrane hydrodynamics and which may have experimental relevance. The framework structure of the theory makes possible its applications to a range of non-equilibrium phenomena in a range of membrane systems, as discussions in the paper of a few limit cases demonstrate.
\end{abstract}

PACS. $05.70 . \mathrm{Np}$ Interface and surface thermodynamics - 83.10-y Fundamentals and theoretical - 82.70-y Disperse systems; complex fluids

\section{Introduction}

Lipid-protein fluid membranes are the most essential structural element in biological cells, defining boundaries of the cells and intracellular organelles. They are also one of the important functional elements, actively participating in many cellular processes. Each biological membrane is composed of a core bilayer of amphiphilic lipids, in which transmembrane proteins are embedded and with which peripheral proteins are associated. In its functional state, the membrane is fluid, allowing the constant movement and organization of the constituent molecules within the structure, and its two-dimensional geometry deforms easily in connection with its function, requiring energies comparable to thermal energy only. Moreover, the membrane constantly exchanges material and energy with its environment; active transport of small solute molecules across the membrane takes place constantly, carried out by membrane proteins that require external energy sources, such as chemical energy provided by ATP, electrochemical energy stored in cross-membrane proton gradients, or light. From the point of view of statistical physics, it is obvious that such a functioning membrane should be treated as a non-equilibrium system and that the dynamics of the membrane is intimately coupled to the dynamics of the bulk fluids within which it is embedded.

\footnotetext{
a e-mail: mlomholt@fysik.sdu.dk

b e-mail: lyngs@memphys.sdu.dk

c e-mail: miao@memphys.sdu.dk; author of correspondence
}

During the last three decades, investigations, characterizations and understanding of the physical properties and behaviour of such membranes and simpler model membranes have fueled both the development of statistical physics of soft condensed matter in general and the development of membrane biophysics in particular, because these systems have posed many issues challenging the traditional framework of statistical physics and also because it has become more and more appreciated that understanding of the physics of the membranes can shed light on their biological functions [1.

For obvious reasons, most of the development of membrane statistical physics has focussed only on the equilibrium, static aspect of the membrane systems. To the purpose of a more complete physical description of biological membranes, however, their non-equilibrium behaviour must be investigated and described. The work presented in this paper is our attempt at taking a step in that direction.

Some recent experimental as well as theoretical studies of simpler model systems of biological membranes have particularly motivated our work [2]. In the model systems, a single type of transmembrane protein, which can be externally driven to actively transport small ions across the membrane, was reconstituted into a core lipid bilayer at various concentrations. By turning on or off the external driving force, the lipid-protein composite membrane was then set in either a non-equilibrium or an equilibrium state, and the non-equilibrium dynamics of the membrane conformation was then investigated experimentally. The- 
ories, in the form of equations of dynamics, were also developed to describe or interpret the experimental observations. The formulation of the theories appears, however, entirely intuition based, which makes its validity rather opaque and its generalization to other lipid-protein composite membranes difficult. We have, therefore, made it our goal to develop a more general and systematic theoretical description of lipid-protein composite membranes in non-equilibrium states, believing that such a theoretical framework will become useful as more and more experimental studies of such systems will be done with their results to be interpreted theoretically.

In fact, a conceptual basis for developing a general description already exists, and it consists of a minimal number of principles of statistical physics: the basic laws of thermodynamics, formulated as a balance equation of entropy, the assumption of local thermodynamic equilibrium in a non-equilibrium system and the dynamic formulation of laws of conservation of mass, momentum and energy, and if relevant, angular momentum. ${ }^{1}$ However, the explicit expressions and implementation of these principles in the case of lipid-protein composite membranes, which in the end result in a set of clearly formulated equations of (hydro)dynamics, must be developed systematically and unambiguously to reflect the physical characteristics pertaining to the membranes.

At the level of continuum descriptions, a membrane is modelled effectively as an interface separating two bulk fluids. Its physical characteristics, however, distinguish it from a simple interface separating two coexisting fluids. Firstly, its molecular composition and material structure differ significantly from those of the two bulk fluids with which it is in contact, and the motion and organization of its constituent molecules within its interface structure provide a set of interesting phenomena. Thus, the material content, and associated with it, the energy content as well as momentum content of the membrane should in general be taken into account. Secondly, its energy (or, thermodynamics) depends not only on its surface area, but also on its local surface geometry, or curvatures 34. Thirdly, its properties with regard to processes of transport of material and energy are in general markedly different from those of the bulk fluids. A good example is the much slower diffusion across the membrane than in the bulk fluids of polar molecules. Thus, the dissipations associated with such transport processes should not be neglected $a$ priori, as is canonically done when non-equilibrium dynamics involving a conventional interface is considered. In fact, there has been experimental evidence suggesting that a membrane should not be considered as dissipationless in its non-equilibrium state [5677. Finally, the typical constituent molecules of a lipid-protein composite membrane - the smaller amphiphilic lipid molecules and much larger transmembrane protein molecules - differ significantly in their molecular chemistry and structures. Consequently, their interactions with the contacting bulk fluids may also

\footnotetext{
1 These conservation laws are sufficient if we limit ourselves to the non-equilibrium dynamics which does not involve nonconserved physical variables.
}

be expected to differ. Our work, in essence, consists in recognizing these unconventional characteristics, clarifying the basic conceptual issues that inevitably rise from considering them, and finally developing an unambiguous description of these characteristics in the context of nonequilibrium dynamics of the system.

To be sure, studies concerning fundamental descriptions of non-equilibrium dynamics of fluid surfaces and interfaces began already three decades ago 89. For example Bedeaux et al. developed for conventional interfaces a formulation of non-equilibrium dynamics by taking into account the energy and momentum content of the interface, from which a connection to equilibrium interface thermodynamics can be made 9 . More recently, a theory for the non-equilibrium dynamics of a two-component surfactant interface separating air and water was presented in the form of a set of dynamic equations, with an emphasis on nonlinear phenomena where phase separation and surface deformations are coupled [10]. In this theory, the mass content and the composition of the interface were explicitly taken into account, and the dependence of the interface thermodynamics on the curvatures was also introduced. The derivation of the dynamic equations was, however, more intuitive than systematic, and certain assumptions were made, which were neither necessary nor justified. Moreover, the transverse transport processes were not discussed. A generalization of this theory to lipid-protein composite membranes did not appear straightforward.

It should be helpful to the reader that we at the outset describe more specifically the system and its non-equilibrium state that we have in mind when developing our theory. The essential picture is sketched out in Fig. 1(a). The system we consider consists of a multi-component membrane of lipid-protein composite, which has a nanometer thickness and which assumes a quasi two-dimensional geometrical shape, and of two aqueous fluids, which the membrane is in contact with. The two fluids can be identical in their chemical compositions and equilibrium thermodynamics, or distinctly different, as in the case of two fluids under conditions of phase separation. In order to keep the theory general, we allow the membrane geometry to be arbitrary, and we do not specify quantitatively the number of molecular species constituting the membrane, nor their exact chemical structures, although the cases where the protein components are transmembrane proteins are of particular interest to us. We also assume that the aqueous fluids may contain more than one types of solute molecules.

We then consider a general non-equilibrium state, where chemical, thermal and mechanical gradients exist both in the directions tangent to the membrane surface and in the direction transverse to the membrane, and where chemical reactions may take place on the membrane. But in the scope of this paper, we will limit ourselves to situations where the length scales over which the assumption of local thermodynamic equilibrium is valid are larger, though not by orders of magnitude, than the thickness of the membrane. The corresponding time scales may be expected to 

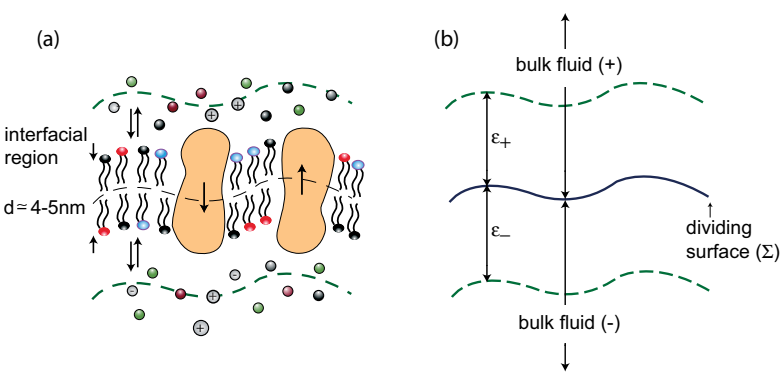

Fig. 1. A schematic sketch of the membrane-fluid system under our consideration. Fig. 1(a) depicts the interfacial region in the real system, showing a membrane composed of bilayerforming lipids and transmembrane proteins in contact with two fluids containing small solutes. Fig. 1(b) illustrates the representation of the interfacial region in the corresponding Gibbs model system.

approach the mesoscopic or above. Given the typical time resolutions of around milliseconds of most experimental techniques used to study membranes, our theory will have a reasonably large range of applicability in terms of length and time scales. It follows then that the membrane is assumed to be in thermodynamic equilibrium with the fluids which it is in immediate contact with. ${ }^{2}$

We would also like to briefly state the philosophy which we have followed when developing the theory. Although the membrane-fluid systems we consider are on microscopic length scales highly inhomogeneous in molecular distributions and transport properties, our theory is not concerned with accurately describing the details of the microscopic-scale inhomogeneity, but aims at describing the inhomogeneity only through those effects that may be observed at mesoscopic or macroscopic scales. Following this philosophy, we have employed an idea originated from Gibbs 11]: the idea of replacing a real, inhomogeneous membrane-fluid system with a model system of two homogeneous bulk fluids separated by an infinitely thin dividing surface and relating to the dividing surface all the excess thermodynamic and hydrodynamic contributions that are required by an equivalence between the thermodynamic and hydrodynamic behaviour of the two systems. This idea is also illustrated graphically by Fig. 1. For later convenience, we will dub this model system "the Gibbs system."

The paper is organized as follows. In Section 2 the necessary surface differential geometry is briefly reviewed. In Section 3 the concept of the surface thermodynamics of the membrane is unambiguously defined based on the idea of the Gibbs model system, and a general expression of the surface thermodynamics is derived and discussed. In addition, a general identity relating different thermodynamic variables associated with the membrane surface is derived, which takes into account the salient mechanical characteristics of membranes. To our knowledge, this

\footnotetext{
${ }^{2}$ Formulation of thermodynamics in situations where this assumption is not valid was first discussed in Ref. [8] in the context of "dynamic surface tension."
}

is the first derivation of such an identity. An assumption made in this Section is that intrinsic orientational degrees of freedom are not relevant. In Section 4 a description of the hydrodynamics of the whole membrane-fluid system is formulated by defining relevant "bulk" and "surface" hydrodynamic variables and by establishing equations of dynamics for all of the hydrodynamic variables based on the fundamental conservation laws. The hydrodynamic description is limited to cases, where systems under consideration have no intrinsic angular momenta. In Section [5] the results from Section 3 and Section 4 combined with the assumption of local thermodynamic equilibrium are used to derive the entropy production, based on which conjugate pairs of general thermodynamic/hydrodynamic force and dissipative current are identified. Constitutive relations for the linear non-equilibrium dynamics of the system are derived in Section 6] In particular, some of these constitutive relations, which under appropriate conditions reduce to those that appear familiar, make predictions about new mechanisms governing various dissipative processes in the membrane. The complete set of constitutive relations, together with the equations of dynamics, thus provide a closed formulation of the hydrodynamics of the whole membrane-fluid system. In Section [7 a number of limit cases of the general theory, which have practical relevance, are discussed, in order that the theory, having been presented in a general and formulistic way, also be seen from a practical point of view. Finally, in the concluding section, Section 8 the theory is discussed within the context of its applications and its connections to experimental measurements. In order that a technical point be made clear, which we expect will often be encountered in applications of the theory, a short appendix is also attached.

\section{Differential geometry of surfaces}

In the continuum theory which we will develop in this paper, a membrane is structurally described as a twodimensional surface. In this section we briefly review, mainly to establish the notation, the mathematical language of two-dimensional differential geometry, which will be used to describe membrane geometry. A more comprehensive introduction can be found in, for instance, Refs. 1213 .

The dynamic shape of the surface is represented by a space-vector function $\boldsymbol{R}=\boldsymbol{R}\left(\xi^{1}, \xi^{2}, t\right)$. The variables $\xi^{1}$ and $\xi^{2}$ are internal coordinates corresponding to a parametrization of the surface and $t$ represents time. At each point on the membrane a basis for three-dimensional vectors can be established. Two of them are the tangential vectors

$$
\boldsymbol{t}_{\alpha} \equiv \partial_{\alpha} \boldsymbol{R} \equiv \frac{\partial \boldsymbol{R}}{\partial \xi^{\alpha}},
$$

where $\alpha=1,2$, and the third is a unit vector normal to the surface,

$$
\boldsymbol{n} \equiv \frac{\partial_{1} \boldsymbol{R} \times \partial_{2} \boldsymbol{R}}{\left|\partial_{1} \boldsymbol{R} \times \partial_{2} \boldsymbol{R}\right|}
$$


The local geometry of the surface is characterized by two surface tensors, the metric tensor and the curvature tensor. The local metric tensor is defined by

$$
g_{\alpha \beta} \equiv \partial_{\alpha} \boldsymbol{R} \cdot \partial_{\beta} \boldsymbol{R} .
$$

It has an inverse, $g^{\alpha \beta}$, which satisfies by definition

$$
g^{\alpha \beta} g_{\beta \gamma}=\delta_{\gamma}^{\alpha}
$$

where $\delta_{\gamma}^{\alpha}$ is the Kronecker delta and where the repeated Greek superscript-subscript indices imply summation following the Einstein summation convention. The metric tensor and its inverse are used to raise and lower Greek indices as in the following example:

$$
\boldsymbol{t}^{\alpha}=g^{\alpha \beta} \boldsymbol{t}_{\beta}, \quad \boldsymbol{t}_{\alpha}=g_{\alpha \beta} \boldsymbol{t}^{\beta} .
$$

The curvature tensor $K_{\alpha \beta}$ is defined via the second derivatives of the surface shape function,

$$
K_{\alpha \beta}=\boldsymbol{n} \cdot \partial_{\alpha} \partial_{\beta} \boldsymbol{R} .
$$

From $K_{\alpha \beta}$ the scalar mean curvature $H$ and Gaussian curvature $K$ can be obtained:

$$
\begin{aligned}
& H=\frac{1}{2} g^{\alpha \beta} K_{\alpha \beta}, \\
& K=\operatorname{det} g^{\alpha \beta} K_{\beta \gamma} .
\end{aligned}
$$

Two other tensors will also be introduced here for later convenience,

$$
\varepsilon_{\alpha \beta} \equiv \epsilon_{\alpha \beta} \sqrt{g}, \quad \quad \varepsilon^{\alpha \beta} \equiv \epsilon^{\alpha \beta} / \sqrt{g},
$$

where $\epsilon_{\alpha \beta}=\epsilon^{\alpha \beta}$ with $\epsilon_{11}=\epsilon_{22}=0$ and $\epsilon_{12}=-\epsilon_{21}=1$ are relative tensors, and $g=\operatorname{det} g_{\alpha \beta}$ is the determinant of the metric tensor.

Expressions of covariant/contravariant differentiations of vector and tensor functions defined on the surfaces are facilitated by the use of the Christoffel symbols, $\Gamma_{\alpha \beta}^{\gamma}$. One instance, which will become particularly useful later, is the covariant differentiation of a surface vector function, $\boldsymbol{w}=w^{\alpha} \boldsymbol{t}_{\alpha}$, given by

$$
D_{\alpha} w^{\beta}=\partial_{\alpha} w^{\beta}+w^{\gamma} \Gamma_{\gamma \alpha}^{\beta} .
$$

The Christoffel symbols can also be defined as certain combinations of the derivatives of the metric tensor, namely,

$$
\Gamma_{\alpha \beta}^{\gamma}=\frac{1}{2} g^{\gamma \delta}\left(\frac{\partial g_{\delta \alpha}}{\partial \xi^{\beta}}+\frac{\partial g_{\beta \delta}}{\partial \xi^{\alpha}}-\frac{\partial g_{\alpha \beta}}{\partial \xi^{\delta}}\right) .
$$

It follows that the covariant divergence of $w^{\alpha}$ can be written as

$$
D_{\alpha} w^{\alpha}=\frac{1}{\sqrt{g}} \partial_{\alpha}\left(\sqrt{g} w^{\alpha}\right) .
$$

Finally, the area of a local differential element of the surface is given by

$$
d A=\sqrt{g} d \xi^{1} d \xi^{2}
$$

an expression which will be repeatedly used in surface integrals.

\section{Membrane thermodynamics}

An important component of a general description of nonequilibrium dynamics of the membrane-fluid system sketched out in Introduction is an appropriate formulation of the equilibrium thermodynamics of the system. In this section we will present such a formulation. Two points which pertain to the system and which have been dealt with in the formulation, may already be mentioned here. Firstly, to describe the thermodynamic effects arising from the microscopic inhomogeneity inherent in the system, we have employed the idea of the Gibbs model system. A subtle issue of principle arises, however, when Gibbs' idea is applied to the membrane system. In the case of a conventional capillary interface, whose mechanical property is entirely described macroscopically by a surface tension experimentally measurable, the position of the Gibbs dividing surface is uniquely determined by the thermodynamic - including mechanical - equivalence between the real and the Gibbs systems 14 15. In the case of the membrane system, whose macroscopic mechanical properties include not only a "membrane tension," but also "membrane bending moments," the thermodynamic equivalence between the real and the Gibbs systems still leaves the position of the Gibbs dividing surface free to be chosen in principle. With a view of making connection to experimental, in particular mechanical, characterizations of membrane systems, where the geometrical profile of a membrane surface is resolved with optical resolutions [16], we do not define quantitatively the position of the theoretical surface, but will work under the assumption that a position can be chosen to be consistent with the experimentally determined one.

Secondly, related to the significance of membrane bending moments in the effective description of a general membrane system 34, the thermodynamic free energies of the membrane systems under our consideration must depend on local geometrical properties such as the principal curvatures. We recognize that, due to such dependence, the free energies no longer scale homogeneously with the size of the membrane and that, consequently, the Gibbs adsorption equation in its canonical form 14 no longer exists. However, a certain statement, in the form of an identity, can still be made about how the different surface thermodynamic variables are related. The derivation of this identity will also be given in this section.

\subsection{The basic equation}

In order to develop a local description of the equilibrium thermodynamics of the membrane system, it is necessary that we consider a small cell, or a volume element, $\bar{\Sigma}$, of the whole membrane-fluid system. A sketch of the cell is given in Fig. 2. The spatial extensions of the cell are defined by a base area element $\Sigma$ on the dividing surface, which spans in a chosen internal coordinate space a fixed rectangle with corners at $\left(\xi^{1}-\Delta \xi^{1} / 2, \xi^{2} \pm \Delta \xi^{2} / 2\right)$ and $\left(\xi^{1}+\Delta \xi^{1} / 2, \xi^{2} \pm \Delta \xi^{2} / 2\right)$, and by a height $\epsilon^{+}$above and a height $\epsilon^{-}$below the dividing surface in the direction of 


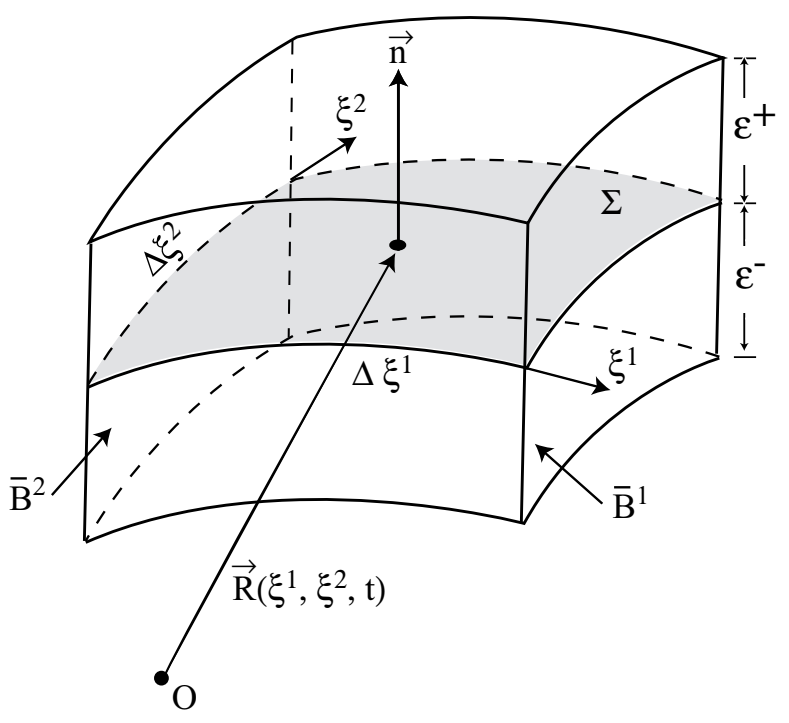

Fig. 2. An illustration of the cell, or volume element, $\bar{\Sigma} . \bar{B}^{1}$ and $\bar{B}^{2}$ refer to the side surfaces of the cell.

the surface normal vector $\boldsymbol{n}$. The local geometry of the surface is described by $\boldsymbol{R}\left(\xi^{1}, \xi^{2}\right)$. $\epsilon^{+}$and $\epsilon^{-}$are chosen such that the physical characteristics of the system at $\epsilon^{+}$ and $\epsilon^{-}$reach those of the two homogeneous bulk fluids. The cell is assumed to be in thermal equilibrium with a uniform temperature, and it is also assumed to be in mechanical equilibrium, although this does not necessarily imply a uniform pressure across the whole cell due to the fact that the membrane interface may be curved. Moreover, in order to include mass motion in the formulation of thermodynamics, the cell is considered to be in uniform motion characterized by a velocity $\boldsymbol{v}$.

Regarding the state of chemical equilibrium in the cell, particular considerations are needed. It is well known that a typical membrane appears impermeable to polar molecules such as small ions on the time scales of seconds 17 and that the reorientations of the constituent amphiphilic lipid and protein molecules from one side of the membrane surface to the other are not observed on similar time scales [18. To take into account this fact, therefore, we introduce an operational definition of species, which is broader than the canonical definition based on the chemical nature of a molecular component: solution molecules of the same chemical structure, but found to be located on the two different sides of the dividing surface will be counted as two different species if their transport across the membrane is a slow process; lipid, or protein molecules having the same chemistry, but oriented in the two opposite directions across the membrane will also be considered as two different species. The equilibrium state which we will develop a thermodynamic description for corresponds to a state where each species is in its chemical equilibrium, but where there is no chemical equilibrium, in the quasistatic sense, between any two species of the same molecular chemistry. To maintain the generality of the theory, we will also allow for in the system the presence of molecular components which do reach chemical equilibrium across the membrane in the same quasi-static sense. Each such component constitutes a single species by our definition.

We denote the energy content in this cell by $E_{\bar{\Sigma}}$. Following the formal structure of thermodynamics, we assume that $E_{\bar{\Sigma}}$ depends on only a few state variables: the molecular numbers of the different species, $N_{A, \bar{\Sigma}}$, where " $A$ " is an index labelling the species; the entropy $S_{\bar{\Sigma}}$; the momentum $\boldsymbol{P}_{\bar{\Sigma}}$; and finally, the variables characterizing the shape and size of the cell, namely, the heights $\epsilon^{ \pm}$, which are assumed to be fixed, and derivatives of the shape field $\boldsymbol{R}\left(\xi^{1}, \xi^{2}\right.$ ) (such as $H$ and $K$ ). ${ }^{3}$ The reason that only derivatives of $\boldsymbol{R}$, not $\boldsymbol{R}$ itself, are allowed is that the thermodynamics should be invariant under rigid translations, provided that the translational-symmetry-breaking effects such as the gravitational force are taken into account as external forces. Based on this assumption, we can therefore express, for the chosen cell, the first and second laws of thermodynamics as a complete differential of $E_{\bar{\Sigma}}$ with respect to all the relevant state variables:

$$
\begin{aligned}
\delta E_{\bar{\Sigma}}=\boldsymbol{v} & \cdot \delta \boldsymbol{P}_{\bar{\Sigma}}+\sum_{A} \mu^{A} \delta N_{A, \bar{\Sigma}}+T \delta S_{\bar{\Sigma}} \\
& -\boldsymbol{F}_{\bar{\Sigma}} \cdot \delta \boldsymbol{R}+\partial_{\alpha}\left(\boldsymbol{S}_{\bar{\Sigma}}^{\alpha} \cdot \delta \boldsymbol{R}\right),
\end{aligned}
$$

where we have already identified the partial derivatives with respect to $\boldsymbol{P}_{\bar{\Sigma}}, N_{A, \bar{\Sigma}}$, and $S_{\bar{\Sigma}}$ with the velocity $\boldsymbol{v}$, the chemical potential for species $A$, and the temperature $T$ of the cell.

In the last two terms, $\boldsymbol{F}_{\bar{\Sigma}}$ is a regular quantity and carries the significance of a physical force, whereas $\boldsymbol{S}_{\bar{\Sigma}}^{\alpha}$ contains differential operators and is related to surface stresses. From a mathematical point view, their presence is not difficult to understand, as they represent the variation in the energy function resulted from any variation in the shape field, $\boldsymbol{R}\left(\xi^{1}, \xi^{2}\right)$, and in turn, variations in its derivatives. Seen from a more physical point of view, the two terms must describe the mechanical work done on the cell when the shape of the dividing surface is changed. To illustrate how their functional forms arise from their mechanical origins is not a trivial issue, and is discussed in a separate paper 20. However, mechanical interpretations of $\boldsymbol{F}_{\bar{\Sigma}}$ and $\boldsymbol{S}_{\bar{\Sigma}}^{\alpha}$ will be made a bit later in the paper, clarifying the reason for expressing the work in those particular functional forms.

To reformulate Eq. (14) by the use of the Gibbs model system, we introduce bulk volume densities for extensive quantities of the cell: $\bar{e}^{ \pm}, \overline{\boldsymbol{p}}^{ \pm}, \bar{s}^{ \pm}$and $\bar{n}_{A}^{ \pm}$. These can be expressed as functions of intensive thermodynamic variables $\boldsymbol{v}, T$ and $\mu^{A}$ 's and are assumed to represent the known thermodynamic behaviour of the homogeneous bulk fluids in the following sense:

$$
\delta \bar{e}^{ \pm}=\boldsymbol{v} \cdot \delta \overline{\boldsymbol{p}}^{ \pm}+T \delta \bar{s}^{ \pm}+\sum_{A} \mu^{A} \delta \bar{n}_{A}^{ \pm}
$$

\footnotetext{
3 In making this assumption, we exclude from our considerations cases where intrinsic orientational degrees of freedom and their independent rotational motion are relevant. An extension which does include those effects is given in Ref. 1920 .
} 
and

$$
\bar{e}^{ \pm}=\boldsymbol{v} \cdot \overline{\boldsymbol{p}}^{ \pm}+T \bar{s}^{ \pm}+\sum_{A} \mu^{A} \bar{n}_{A}^{ \pm}-p^{ \pm},
$$

where $p^{ \pm}$denotes the bulk function of velocity, temperature and chemical potentials that corresponds to pressure.

We can now define the excess area densities of extensive quantities, which must be associated with the dividing surface according to the construction of the Gibbs model system:

$$
\begin{aligned}
e & =\frac{1}{A_{\Sigma}}\left(E_{\bar{\Sigma}}-V_{\bar{\Sigma}}^{+} \bar{e}^{+}-V_{\bar{\Sigma}}^{-} \bar{e}^{-}\right), \\
\boldsymbol{p} & =\frac{1}{A_{\Sigma}}\left(\boldsymbol{P}_{\bar{\Sigma}}-V_{\tilde{\Sigma}}^{+} \overline{\boldsymbol{p}}^{+}-V_{\bar{\Sigma}}^{-} \overline{\boldsymbol{p}}^{-}\right), \\
s & =\frac{1}{A_{\Sigma}}\left(S_{\bar{\Sigma}}-V_{\bar{\Sigma}}^{+} \bar{s}^{+}-V_{\bar{\Sigma}}^{-} \bar{s}^{-}\right), \\
n_{A} & =\frac{1}{A_{\Sigma}}\left(N_{A, \bar{\Sigma}}-V_{\bar{\Sigma}}^{+} \bar{n}_{A}^{+}-V_{\bar{\Sigma}}^{-} \bar{n}_{A}^{-}\right),
\end{aligned}
$$

where $A_{\Sigma}$ is the area of the surface element $\Sigma$, and $V_{\bar{\Sigma}}^{ \pm}$ represents the volume of the cell part which is above/below the dividing surface, respectively. It follows that

$$
\boldsymbol{p}=\rho \boldsymbol{v},
$$

where $\rho=\sum_{A} m^{A} n_{A}$ is the excess mass density.

Using these excess quantities, together with

$$
\begin{aligned}
& V_{\bar{\Sigma}}^{ \pm}=A_{\Sigma}\left(\epsilon^{ \pm} \mp\left(\epsilon^{ \pm}\right)^{2} H+\frac{1}{3}\left(\epsilon^{ \pm}\right)^{3} K\right), \\
& A_{\Sigma}=\Delta \xi^{1} \Delta \xi^{2} \sqrt{g}
\end{aligned}
$$

where we have assumed that the radii of curvature are bigger than $\epsilon^{ \pm}$, we finally arrive at the following reformulation of Eq. (14),

$$
\begin{aligned}
\delta(\sqrt{g} e)= & \boldsymbol{v} \cdot \delta(\sqrt{g} \boldsymbol{p})+T \delta(\sqrt{g} s)+\sum_{A} \mu^{A} \delta\left(\sqrt{g} n_{A}\right) \\
& -\sqrt{g} \boldsymbol{f}_{\mathrm{rs}} \cdot \delta \boldsymbol{R}+\sqrt{g} D_{\alpha}\left(\boldsymbol{S}^{\alpha} \cdot \delta \boldsymbol{R}\right)
\end{aligned}
$$

Vector quantities $\boldsymbol{f}_{\mathrm{rs}}$ and $\boldsymbol{S}^{\alpha}$ are defined by

$$
\begin{aligned}
\boldsymbol{f}_{\mathrm{rs}} & \equiv \frac{\boldsymbol{F}_{\bar{\Sigma}}}{A_{\Sigma}}-D_{\alpha} \boldsymbol{B}_{+}^{\alpha}-D_{\alpha} \boldsymbol{B}_{-}^{\alpha} \\
\boldsymbol{S}^{\alpha} & \equiv \frac{1}{A_{\Sigma}} \boldsymbol{S}_{\bar{\Sigma}}^{\alpha}-\boldsymbol{B}_{+}^{\alpha}-\boldsymbol{B}_{-}^{\alpha}+\boldsymbol{n}\left(C_{+}^{\alpha \beta}+C_{-}^{\alpha \beta}\right) \partial_{\beta},
\end{aligned}
$$

where

$$
\begin{aligned}
\boldsymbol{B}_{ \pm}^{\alpha} \equiv & -\epsilon^{ \pm} p^{ \pm} \boldsymbol{t}^{\alpha} \pm p^{ \pm}\left(\epsilon^{ \pm}\right)^{2}\left(H g^{\alpha \beta}-\frac{1}{2} K^{\alpha \beta}\right) \boldsymbol{t}_{\beta} \\
& -D_{\beta} C_{ \pm}^{\alpha \beta} \boldsymbol{n} \\
C_{ \pm}^{\alpha \beta} \equiv & \mp\left(\epsilon^{ \pm}\right)^{2} p^{ \pm} \frac{1}{2} g^{\alpha \beta}+\frac{1}{3}\left(\epsilon^{ \pm}\right)^{3} p^{ \pm}\left(2 H g^{\alpha \beta}-K^{\alpha \beta}\right) .
\end{aligned}
$$

Note that the physics represented by Eq. (24) should be independent of any specific numerical values of $\epsilon^{ \pm}$.
Based on Eq. (24) the mathematical area element $\Sigma$ on the dividing surface may be viewed as an effective surface system which has its own thermodynamic properties and which interacts with its "surroundings." In this effective picture, the last two terms in Eq. (24) - related to the mechanical-work terms in Eq. (14) - may be interpreted as the work done on the effective surface system by its surroundings: In Section 5, it will become clear that $\boldsymbol{f}_{\mathrm{rs}}$ must balance the effective force exerted on $\Sigma$ by sources external to the dividing surface under mechanical equilibrium. Similarly, $\sqrt{g} D_{\alpha}\left(\boldsymbol{S}^{\alpha} \cdot \delta \boldsymbol{R}\right)$ will be shown in Eq. (69) to represent the work done on $\Sigma$ by the rest of the dividing surface. But, this interpretation can already be made plausible here by noting that $\int_{\Sigma} d^{2} \xi \sqrt{g} D_{\alpha}\left(\boldsymbol{S}^{\alpha} \cdot \delta \boldsymbol{R}\right)$ can actually be rewritten as an integral over the boundary $\partial \Sigma$ of the area element $\Sigma$

$$
\int_{\Sigma} d^{2} \xi \sqrt{g} D_{\alpha}\left(\boldsymbol{S}^{\alpha} \cdot \delta \boldsymbol{R}\right)=\int_{\partial \Sigma} d s \nu_{\alpha} \boldsymbol{S}^{\alpha} \cdot \delta \boldsymbol{R},
$$

where $s$ is the arc length and $\nu_{\alpha} t^{\alpha}$ is a unit normal vector pointing away from the boundary $\partial \Sigma$.

There is in fact a connection between $\boldsymbol{f}_{\mathrm{rs}}$ and $\boldsymbol{S}^{\alpha}$. If $\boldsymbol{S}_{(0)}^{\alpha}$ is used to represent all the contributions in $\boldsymbol{S}^{\alpha}$ that do not involve differential operators, then it can be seen from Eq. (24) that the following relationship must be satisfied, as a consequence of the invariance of the thermodynamics under rigid translations:

$$
\boldsymbol{f}_{\mathrm{rs}}=D_{\alpha} \boldsymbol{S}_{(0)}^{\alpha} .
$$

$f_{\mathrm{rs}}$ accounts for the total mechanical force exerted on the effective surface element $\Sigma$ by the rest of the effective surface. In what follows, $\boldsymbol{f}_{\mathrm{rs}}$ will be referred to as the "restoring force." It is clear that $\boldsymbol{S}_{(0)}^{\alpha}$ should be identified as the surface stress tensor as defined in 2122 .

The above interpretations make clear the reason for organizing the geometry-dependent work explicitly into the two particular functional terms in Eq. (14). We would like to note also that these interpretations can be obtained in a more physically intuitive and direct way by formulating the mechanical work explicitly, once a model of the mechanical behaviour of the inhomogeneous cell is given. $\boldsymbol{F}_{\bar{\Sigma}}$ and $\boldsymbol{S}_{\bar{\Sigma}}^{\alpha}$, or $\boldsymbol{f}_{\mathrm{rs}}$ and $\boldsymbol{S}^{\alpha}$, can be identified with the mechanical model quantities. But, we defer the discussion of that topic to another paper [20.

It can be seen easily that Eq. (24) does not define $\boldsymbol{S}^{\alpha}$ uniquely. An addition to it of the following type, for instance,

$$
\varepsilon^{\alpha \beta}\left(\partial_{\beta} \boldsymbol{V}+\boldsymbol{V} \partial_{\beta}\right)
$$

where $\boldsymbol{V}$ is an arbitrary vector, does not change the mechanical work at all. This seemingly mathematical point implies in fact a non-trivial physical statement: the theoretical characterization of the mechanical behaviour of a membrane system in terms of an effective surface stress tensor and surface bending-moment tensor is not unique, as opposed to the "belief" implied in the canonical description of membrane mechanics 23. We will discuss and clarify this issue elsewhere [20].

Eq. (24) thus provides the basic equation of the membrane surface thermodynamics and will be used later in 
our description of non-equilibrium dynamics of the membrane system. Once an explicit functional form of $e$ and the values of the surface thermodynamic variables, $\boldsymbol{p}, s$, $n_{A}$ 's, and $\boldsymbol{R}$ are assumed to be known, Eq. (24), or its integrated form $E=\int_{\mathrm{M}} d A e$, can be used to determine the other physically measurable thermodynamic variables of the surface as follows:

$$
\begin{aligned}
\boldsymbol{v} & =\left.\frac{1}{\sqrt{g}} \frac{\delta E}{\delta \boldsymbol{p}}\right|_{\boldsymbol{R},\left\{n_{A}\right\}, s}, \\
T & =\left.\frac{1}{\sqrt{g}} \frac{\delta E}{\delta s}\right|_{\boldsymbol{p}, \boldsymbol{R},\left\{n_{A}\right\}}, \\
\boldsymbol{f}_{\mathrm{rs}} & =-\left.\frac{1}{\sqrt{g}} \frac{\delta E}{\delta \boldsymbol{R}}\right|_{\sqrt{g} \boldsymbol{p}, \sqrt{g} s,\left\{\sqrt{g} n_{A}\right\}}, \\
\mu^{A} & =\left.\frac{1}{\sqrt{g}} \frac{\delta E}{\delta n_{A}}\right|_{\boldsymbol{p}, s, \boldsymbol{R},\left\{n_{B} \mid B \neq A\right\}}
\end{aligned}
$$

\subsection{A useful identity derived from reparametrization invariance}

An important element of the canonical thermodynamics of a capillary interface between two coexisting fluids is the so-called Gibbs adsorption equation [14, which relates together the different intensive thermodynamic variables defining the state of the interface. It results from the fact that the excess thermodynamic free energies associated with such an interface scale proportionally with the area of the interface at constant excess densities of the extensive quantities, or equivalently, that the mechanical characterization of the interface is given by a single intensive quantity, the surface tension.

The thermodynamics of the type of membrane systems under our considerations is, however, different. The canonical model of the membrane mechanics proposed by Helfrich and Evans provides an example. In the model, the part of the free energy associated with the membrane mechanics is given by $\int_{\Sigma} d A\left(2 \kappa H^{2}+\sigma_{0}\right)$, where $\kappa$ and $\sigma_{0}$ are constants. It is clear that, if the linear size of the membrane surface is scaled by $\boldsymbol{R} \rightarrow \lambda \boldsymbol{R}$, the term involving $\sigma_{0}$ will increase with $\lambda^{2}$ while the one including $\kappa$ (the bending term) will not change. Consequently, the Gibbs adsorption equation no longer exists; and the concept of surface tension alone is no longer sufficient to describe the mechanical behaviour of the membrane surface at mesoscopic or macroscopic scales. Instead, as Eq. (24) implies, the restoring force $\boldsymbol{f}_{\mathrm{rs}}$ provides an appropriate mechanical quantity.

It is obvious from Eq. (24) that $\boldsymbol{f}_{\text {rs }}$ depends on the determining (surface) thermodynamic variables such as $\boldsymbol{v}$, $T, \mu^{A}$ 's as well as the geometry of the dividing surface. It turns out that the tangential components of $\boldsymbol{f}_{\mathrm{rs}}$ are intimately connected with the spatial inhomogeneities in $\boldsymbol{v}, T$, and $\mu^{A}$ 's. This connection arises from the fact that both the thermodynamic and the hydrodynamic behaviour of the dividing surface is that of a two-dimensional "fluid system." In other words, they should remain invariant under any change of the internal coordinate system.
To derive the explicit expression of the connection, we consider a situation where there exist spatial inhomogeneities in the surface thermodynamic variables. The total excess energy $E$ associated with the dividing surface is then the surface integral of the local density $e$, i.e. a functional of $\boldsymbol{p}, s, n_{A}$ 's and $\boldsymbol{R}$. Under an arbitrary, infinitesimal change of internal coordinates, or "reparametrization" of the dividing surface,

$$
\xi^{\prime \alpha}=\xi^{\alpha}+\delta \xi^{\alpha}
$$

the functional form of the local density of excess entropy, as an example of a physical quantity, must change from $s\left(\xi^{1}, \xi^{2}\right)$ in the old coordinate system to another form $s^{\prime}\left(\xi^{\prime 1}, \xi^{\prime 2}\right)$ in the new such that

$$
s\left(\xi^{1}, \xi^{2}\right)=s^{\prime}\left(\xi^{\prime 1}, \xi^{2}\right)=s^{\prime}\left(\xi^{1}, \xi^{2}\right)+\delta \xi^{\alpha} \partial_{\alpha} s
$$

as required by the reparametrization invariance. This is equivalent to making the following variation in the functional form of the entropy density expressed in the old coordinate system:

$$
\delta s \equiv s^{\prime}\left(\xi^{1}, \xi^{2}\right)-s\left(\xi^{1}, \xi^{2}\right)=-\delta \xi^{\alpha} \partial_{\alpha} s .
$$

The variations in the functional forms of $\boldsymbol{p}, \boldsymbol{R}$ and $n_{A}$ can be expressed similarly.

Under fixed boundary conditions, the above variations lead to a variation in the integrated energy $E$

$$
\begin{aligned}
\delta E=\int_{\mathrm{M}} d^{2} \xi & (\boldsymbol{v} \cdot \delta(\sqrt{g} \boldsymbol{p})+T \delta(\sqrt{g} s) \\
& \left.-\sqrt{g} \boldsymbol{f}_{\mathrm{rs}} \cdot \delta \boldsymbol{R}+\sum_{A} \mu^{A} \delta\left(\sqrt{g} n_{A}\right)\right),
\end{aligned}
$$

where Eq. (24) has been used. Obviously, this variation must be zero.

Inserting into Eq. (36) Eq. (35), its analogs for $\boldsymbol{p}, n_{A}$ 's and $\boldsymbol{R}$, as well as $\delta(\sqrt{g})=\sqrt{g} \boldsymbol{t}^{\alpha} \cdot \partial_{\alpha} \delta \boldsymbol{R}$, and performing a partial integration yields

$$
\begin{aligned}
\delta E=\int_{\mathrm{M}} d A & \left(\boldsymbol{p} \cdot \partial_{\alpha} \boldsymbol{v}+s \partial_{\alpha} T\right. \\
& \left.+\boldsymbol{f}_{\mathrm{rs}} \cdot \boldsymbol{t}_{\alpha}+\sum_{A} n_{A} \partial_{\alpha} \mu^{A}\right) \delta \xi^{\alpha}=0 .
\end{aligned}
$$

Since $\delta \xi^{\alpha}$ is arbitrary, it can finally be concluded that

$$
\boldsymbol{f}_{\mathrm{rs}} \cdot \boldsymbol{t}_{\alpha}+\boldsymbol{p} \cdot \partial_{\alpha} \boldsymbol{v}+s \partial_{\alpha} T+\sum_{A} n_{A} \partial_{\alpha} \mu^{A}=0
$$

must always hold. This identity will become a useful one in the formulation of non-equilibrium dynamics.

Two remarks are worth making here. First, although the physical reason underlying the above identity appears conceptually obvious, we are not aware of any earlier work where the result has been systematically derived. Secondly, although we have made the derivation with membrane systems in mind, the result also applies to a conventional capillary interface with a spatially varying surface 
tension $\sigma$, in which case $\boldsymbol{f}_{\mathrm{rs}} \cdot \boldsymbol{t}_{\alpha}=\partial_{\alpha} \sigma$. Eq. (38) thus coincides with the expression of the Gibbs adsorption equation when it is applied to cases where spatial inhomogeneities in the interface are present [14].

\section{Dynamic Formulation of Conservation Laws}

Having formulated a description of local thermodynamic equilibrium of the membrane-fluid system in terms of the surface excess quantities, we now proceed to consider the general non-equilibrium state of the system as defined in Introduction and develop a theory which describes the dynamics of the non-equilibrium state. Following the philosophy of developing an effective theory by the use of the Gibbs model system, where two bulk fluids meet at an infinitely thin dividing surface, we assume that the hydrodynamic description of the two bulk fluids, in terms of their local thermodynamics and transport properties, is entirely known, and thus we relate, not only the thermodynamic, but also the hydrodynamic, effects associated with the microscopic inhomogeneity in the real system to the dividing surface and to its modes of interaction with the two bulk fluids.

To make the presentation easy to follow, we first define a few notations pertaining to the description of the space. Similar to previous notation, $\boldsymbol{R}\left(\xi^{1}, \xi^{2}, t\right)$ is used to represent the dynamic shape of the dividing surface, and the space is then divided into two regions separated by the dividing surface: "+"-region refers to the bulk-fluid region which the normal of the surface, $\boldsymbol{n}\left(\xi^{1}, \xi^{2}, t\right)$ points into and "-"-region the other. A scalar function $f(\boldsymbol{r}, t)$ is introduced such that it is zero on the dividing surface and positive/negative on the $+/-$ side, respectively; two Heaviside step functions are then defined as $\theta^{ \pm}(\boldsymbol{r}, t)=\theta( \pm f(\boldsymbol{r}, t))$. A few identities follow immediately,

$$
\begin{aligned}
& \partial_{t} \theta^{ \pm}=\mp \int_{\mathrm{M}} d A \boldsymbol{n} \cdot \partial_{t} \boldsymbol{R} \delta(\boldsymbol{r}-\boldsymbol{R}), \\
& \boldsymbol{\nabla} \theta^{ \pm}= \pm \int_{\mathrm{M}} d A \boldsymbol{n} \delta(\boldsymbol{r}-\boldsymbol{R}), \\
& \boldsymbol{t}_{\alpha} \cdot \boldsymbol{\nabla} \delta(\boldsymbol{r}-\boldsymbol{R})=-\partial_{\alpha} \delta(\boldsymbol{r}-\boldsymbol{R}),
\end{aligned}
$$

which will be used below.

The starting point of the hydrodynamic description is a formulation of the basic laws of conservation of the molecular number of each species, momentum and energy for the model system in the context of non-equilibrium dynamics. Specifically, it is assumed that the following equation of dynamics holds,

$$
\frac{\partial}{\partial t} \bar{x}(\boldsymbol{r}, t)=-\boldsymbol{\nabla} \cdot \overline{\boldsymbol{J}}_{X}(\boldsymbol{r}, t)+\bar{\sigma}_{x}
$$

where $\bar{x}(\boldsymbol{r}, t)$ represents the volume density of a conserved quantity $X$ and runs over the number density of a species, $\bar{n}_{A}(\boldsymbol{r}, t)$, the momentum density, $\overline{\boldsymbol{p}}(\boldsymbol{r}, t)$, and the energy density $\bar{e}(\boldsymbol{r}, t)$, and where $\overline{\boldsymbol{J}}_{X}(\boldsymbol{r}, t)$ represents the corresponding flux. This formulation is broad in that it allows for the presence of a term $\bar{\sigma}_{x}$, which can account for "sinksource" mechanisms in the dynamics of conserved quantities. For example, when $X$ represent molecular numbers, $\bar{\sigma}_{x}$ can be used to describe the kinetics of chemical reactions. In the case where an electric field $\boldsymbol{E}$ is applied on the system, which may contain molecules carrying charges $\left\{q^{A}\right\}, \overline{\boldsymbol{\sigma}}_{p}=\boldsymbol{E} \sum_{A} q^{A} \bar{n}_{A}$ and $\bar{\sigma}_{e}=\sum_{A} q^{A} \bar{n}_{A} \boldsymbol{E} \cdot \boldsymbol{v}_{A}$ may be used to model the effects of the electric field, where $\boldsymbol{v}_{A}$ denotes the velocity of species $A$.

In principle, the law of angular-momentum conservation should also be included. In standard hydrodynamic descriptions of conventional fluids, the canonical approximation is that each local fluid element has no intrinsic angular momentum. In the case of the type of membranefluid systems under our considerations, it would be expected that physical situations exist where the approximation is a valid one, and also that in other situations it no longer holds. But, we will work with the simpler cases where the approximation may be made.

Based on the structure of the model system, $\bar{x}(\boldsymbol{r}, t)$ is expressed as

$$
\begin{aligned}
& \bar{x}(\boldsymbol{r}, t)=\bar{x}^{+}(\boldsymbol{r}, t) \theta^{+}(\boldsymbol{r}, t)+\bar{x}^{-}(\boldsymbol{r}, t) \theta^{-}(\boldsymbol{r}, t) \\
& +\int_{\mathrm{M}} d A\left(\xi^{1}, \xi^{2}, t\right) x\left(\xi^{1}, \xi^{2}, t\right) \delta\left(\boldsymbol{r}-\boldsymbol{R}\left(\xi^{1}, \xi^{2}, t\right)\right),
\end{aligned}
$$

where $\bar{x}^{ \pm}(\boldsymbol{r}, t)$ represents the volume density of $X$ in the " \pm "-bulk fluid, M indicates that the surface integral is over the whole dividing surface, and finally, $x\left(\xi^{1}, \xi^{2}, t\right)$ is the surface density of the excess of quantity $X$. It follows immediately from the concept of the model system and the assumption of local thermodynamic equilibrium that $\bar{n}_{A}^{ \pm}(\boldsymbol{r}, t)^{\prime}$ 's, $\overline{\boldsymbol{p}}^{ \pm}(\boldsymbol{r}, t), \bar{e}^{ \pm}(\boldsymbol{r}, t)$ and $\bar{s}^{ \pm}(\boldsymbol{r}, t)$ satisfy the expressions of the bulk thermodynamics, Eq. (15) and Eq. (16). Thus, the two equations provide operational definitions of the corresponding chemical-potential fields, $\bar{\mu}_{ \pm}^{A}(\boldsymbol{r}, t)$ 's, hydrodynamic velocity fields $\overline{\boldsymbol{v}}^{ \pm}(\boldsymbol{r}, t)$, temperature fields, $\bar{T}^{ \pm}(\boldsymbol{r}, t)$, and pressure fields $\bar{p}^{ \pm}(\boldsymbol{r}, t)$. Regarding the thermodynamic characterization of the dividing surface, a similar assumption is made: the non-equilibrium surface density of the excess energy, $e\left(\xi^{1}, \xi^{2}, t\right)$, is still functionally related to the other surface densities, $\boldsymbol{p}\left(\xi^{1}, \xi^{2}, t\right), s\left(\xi^{1}, \xi^{2}, t\right)$, and $n_{A}\left(\xi^{1}, \xi^{2}, t\right)$ 's as well as the shape field $\boldsymbol{R}\left(\xi^{1}, \xi^{2}, t\right)$ according to Eq. (24). The conjugate variables defined thus by Eq. (32), $\boldsymbol{v}\left(\xi^{1}, \xi^{2}, t\right)$, $T\left(\xi^{1}, \xi^{2}, t\right)$, and $\mu^{A}\left(\xi^{1}, \xi^{2}, t\right)$ 's are then considered as the velocity, the temperature and the chemical potentials of the dividing surface, respectively, and $\boldsymbol{f}_{\mathrm{rs}}\left(\xi^{1}, \xi^{2}, t\right)$ can be identified with the mechanical force exerted on the dividing surface. Consequently, Eq. (21) and Eq. (38) also hold.

Similar to that of $\bar{x}(\boldsymbol{r}, t)$, the model expression of the corresponding flux $\overline{\boldsymbol{J}}_{X}(\boldsymbol{r}, t)$ is given by

$$
\begin{aligned}
& \overline{\boldsymbol{J}}_{X}(\boldsymbol{r}, t)=\overline{\boldsymbol{J}}_{X}^{+}(\boldsymbol{r}, t) \theta^{+}(\boldsymbol{r}, t)+\overline{\boldsymbol{J}}_{X}^{-}(\boldsymbol{r}, t) \theta^{-}(\boldsymbol{r}, t) \\
& +\int_{\mathrm{M}} d A\left[\boldsymbol{j}_{x}^{(0)}\left(\xi^{1}, \xi^{2}, t\right)\right. \\
& \left.\quad-\boldsymbol{j}_{x}^{(1)}\left(\xi^{1}, \xi^{2}, t\right)(\boldsymbol{n} \cdot \boldsymbol{\nabla})\right] \delta(\boldsymbol{r}-\boldsymbol{R}),
\end{aligned}
$$


where $\overline{\boldsymbol{J}}_{X}^{ \pm}(\boldsymbol{r}, t)$ represents the flux in the " \pm "-bulk fluid. It is one of the model statements that the functional dependence of $\overline{\boldsymbol{J}}_{X}^{ \pm}(\boldsymbol{r}, t)$ on the relevant bulk hydrodynamic state variables and bulk transport coefficients is known. Quantities $\boldsymbol{j}_{x}^{(0)}\left(\xi^{1}, \xi^{2}, t\right)$ and $\boldsymbol{j}_{x}^{(1)}\left(\xi^{1}, \xi^{2}, t\right)$ account for the excess contributions due to the inhomogeneity in the real system.

The presence of a non-zero $\boldsymbol{j}_{x}^{(1)}\left(\xi^{1}, \xi^{2}, t\right)$ in the above model expression is only necessary when $X$ represents linear momentum. In that case $\boldsymbol{j}_{p}^{(1)}\left(\xi^{1}, \xi^{2}, t\right)$ can be identified as the surface flux of angular momentum. It includes the contributions from both the motion and the mechanical stress in the material, and is non-zero even when there is no motion. The reason for it is that a complete mechanical characterization of a membrane within the Gibbs model system requires both an effective surface-stress tensor, included in $\boldsymbol{j}_{p}^{(0)}\left(\xi^{1}, \xi^{2}, t\right)$, and an effective, non-zero bendingmoment tensor, represented by a non-zero $\boldsymbol{j}_{p}^{(1)}\left(\xi^{1}, \xi^{2}, t\right)$, in order that there is a mechanical equivalence between the real and the model systems when their respective distributions of mechanical properties are integrated over the transverse dimension across the inhomogeneous region. A more detailed discussion of this issue will be given in Ref. 20.

Inserting the model expressions, Eq. (41) and Eq. (42), in the conservation law, Eq. (40), carrying out the partial differentiations using Eq. (39) and performing some partial integrations lead to the following set of equations:

$$
\begin{aligned}
\frac{\partial}{\partial t} \bar{x}^{ \pm}= & -\boldsymbol{\nabla} \cdot \overline{\boldsymbol{J}}_{X}^{ \pm}+\bar{\sigma}_{x}^{ \pm} \\
D_{t} x= & -D_{\alpha}\left[\left(\boldsymbol{j}_{x}^{(0)}-x \partial_{t} \boldsymbol{R}\right) \cdot \boldsymbol{t}^{\alpha}+\boldsymbol{j}_{x}^{(1)} \cdot \boldsymbol{t}_{\beta} K^{\alpha \beta}\right] \\
& -\left(\boldsymbol{J}_{X}^{+} \cdot \boldsymbol{n}-x^{+} \partial_{t} \boldsymbol{R} \cdot \boldsymbol{n}\right) \\
& +\left(\boldsymbol{J}_{X}^{-} \cdot \boldsymbol{n}-x^{-} \partial_{t} \boldsymbol{R} \cdot \boldsymbol{n}\right)+\sigma_{x} \\
0= & -\left(\boldsymbol{j}_{x}^{(0)}-x \partial_{t} \boldsymbol{R}\right) \cdot \boldsymbol{n}+D_{\alpha}\left(\boldsymbol{j}_{x}^{(1)} \cdot \boldsymbol{t}^{\alpha}\right), \\
0= & \boldsymbol{j}_{x}^{(1)} \cdot \boldsymbol{n},
\end{aligned}
$$

where a new differential operator with respect to $t$, $D_{t}(\bullet) \equiv 1 / \sqrt{g} \partial_{t}(\sqrt{g} \bullet)$, has been introduced. $\boldsymbol{J}_{X}^{ \pm}$and $x^{ \pm}$ represent the boundary values of the bulk hydrodynamic quantities, i.e., the values of $\overline{\boldsymbol{J}}^{ \pm}(\boldsymbol{r}, t)$ and $\bar{x}^{ \pm}(\boldsymbol{r}, t)$ evaluated at the dividing surface, respectively. $\sigma_{x}$ is the surface excess of the rate of generation/disappearance of quantity $X$ associated with the sink-source term in Eq. (40).

Eq. (43) is a reiteration of one of the model statements that fluids with the known bulk behaviours fill the regions on the two sides of the infinitely thin dividing surface. Eq. (44) represents the set of equations governing the dynamics of the relevant excess surface quantities. Eq. 45) and Eq. (46) are simply conditions of self-consistency, implied in the Gibbs-model construction, on the normal components of $\boldsymbol{j}_{x}^{(0)}$ and $\boldsymbol{j}_{x}^{(1)}$.

Based on Eq. (44), a tangential current within the dividing surface, denoted by $j_{x}^{\alpha}$, and two transverse currents entering/leaving the dividing surface, denoted by $j_{x}^{ \pm}$, can be identified:

$$
\begin{aligned}
& j_{x}^{\alpha} \equiv\left(\boldsymbol{j}_{x}^{(0)}-x \partial_{t} \boldsymbol{R}\right) \cdot \boldsymbol{t}^{\alpha}+\boldsymbol{j}_{x}^{(1)} \cdot \boldsymbol{t}_{\beta} K^{\alpha \beta}, \\
& j_{x}^{ \pm} \equiv \boldsymbol{J}_{X}^{ \pm} \cdot \boldsymbol{n}-x^{ \pm} \partial_{t} \boldsymbol{R} \cdot \boldsymbol{n} .
\end{aligned}
$$

Eq. (44) can thus be written as

$$
\begin{aligned}
D_{t} e & =-D_{\alpha} j_{e}^{\alpha}+j_{e}^{-}-j_{e}^{+}, \\
D_{t} \boldsymbol{p} & =-D_{\alpha} \boldsymbol{j}_{p}^{\alpha}+\boldsymbol{j}_{p}^{-}-\boldsymbol{j}_{p}^{+}, \\
D_{t} n_{A} & =-D_{\alpha} j_{A}^{\alpha}+j_{A}^{-}-j_{A}^{+}+\sum_{K} \nu_{A, K} \xi^{K},
\end{aligned}
$$

for cases where $\sigma_{p}=\sigma_{e}=0$. We will only consider such cases in what follows.

The last term in Eq. (51) has been added to allow for the possibility of "chemical reactions" taking place in the membrane. The summation index $K$ runs over all possible reactions, $\xi^{K}$ is the rate of reaction $K$ per unit area and $\nu_{A, K}$ the stoichiometric coefficient of species $A$ in reaction $K$. The word "chemical reaction" in our theory should be understood in a broader sense than that pertaining to a genuine chemical reaction. Connected to the assumption used in the formulation of thermodynamics that the membrane is considered to be quasi-statically impermeable to certain chemical species, non-equilibrium transport across the membrane of any of those chemical species (denoted by $\bar{C}$ ), such as the flip-flop process of a particular lipid species, is modelled by the following "reaction,"

$$
\bar{C}^{+} \rightleftarrows \bar{C}^{-},
$$

where $\bar{C}^{+}$and $\bar{C}^{-}$are considered as two different labelled species.

It must have not escaped the reader that Eq. (41) and Eq. (42) on their own are not sufficient to define $x\left(\xi^{1}, \xi^{2}, t\right), \boldsymbol{j}_{x}^{(0)}\left(\xi^{1}, \xi^{2}, t\right)$ and $\boldsymbol{j}_{x}^{(1)}\left(\xi^{1}, \xi^{2}, t\right)$. At the conceptual level, the definitions of the surface excess quantities may be understood in the following sense. Consider the cell $\bar{\Sigma}$ defined in Section 3 and bear in mind in particular that $\epsilon^{ \pm}$must be chosen such that the hydrodynamic behaviour of the real system coincides with that of the model system outside the top and bottom surfaces of the cell. If $X_{\bar{\Sigma}}$ denotes the amount of quantity $X$ in the cell in the real system, the following condition of equivalence between the real and the model systems

$$
\int_{\bar{\Sigma}} d V \bar{x}(\boldsymbol{r}, t)=X_{\bar{\Sigma}}
$$

then defines $x\left(\xi^{1}, \xi^{2}, t\right)$. In a similar fashion, a number of conditions of equivalence should be satisfied by the model quantities $\boldsymbol{j}_{x}^{(0)}\left(\xi^{1}, \xi^{2}, t\right)$ and $\boldsymbol{j}_{x}^{(1)}\left(\xi^{1}, \xi^{2}, t\right)$. If $\bar{B}^{\alpha}$ represents the cross section of $\bar{\Sigma}$ at constant $\xi^{\alpha}$, then the following model quantity

$$
\int_{\bar{B}^{\alpha}} d \overline{\boldsymbol{A}} \cdot\left[\overline{\boldsymbol{J}}_{X}-\bar{x} \partial_{t}(\boldsymbol{R}+h \boldsymbol{n})\right],
$$

where $d \overline{\boldsymbol{A}}$ is a normally directed area element on $\bar{B}^{\alpha}$ and where the integration is taken over the transverse dimension, $h$, along the normal $\boldsymbol{n}$, should equal to the total current crossing $\bar{B}^{\alpha}$ in the real system. 
The discussion in the preceding paragraph in fact gives rise to a subtle issue concerning the relationship between the surface density of the excess momentum, $\boldsymbol{p}$, defined by Eq. (53), and the excess current associated with the total mass, $\boldsymbol{j}_{\rho}^{(0)}$. Given $\boldsymbol{p}$, the hydrodynamic velocity $\boldsymbol{v}$ associated with the dividing surface is determined by the local thermodynamics, i.e.,

$$
\boldsymbol{v}=\boldsymbol{p} / \rho
$$

where $\rho$ is the surface density of the excess mass. $\boldsymbol{j}_{\rho}^{(0)}$, defined by the condition Eq. (54), is not equal to $\boldsymbol{p}$ in principle. ${ }^{4}$ The difference is on the order of $\epsilon^{ \pm} / R$ where $R$ is curvature, and it will be neglected, to a first approximation. In what follows, we will thus use

$$
\boldsymbol{j}_{\rho}^{(0)}=\rho \boldsymbol{v}
$$

By this approximation, Eq. (45) reduces to

$$
\boldsymbol{v} \cdot \boldsymbol{n}=\partial_{t} \boldsymbol{R} \cdot \boldsymbol{n}
$$

a description consistent with our intuition. An alternative expression of Eq. (57), which will also be used later, is

$$
\boldsymbol{v}=v^{\alpha} \boldsymbol{t}_{\alpha}+\partial_{t} \boldsymbol{R}
$$

where $v^{\alpha} \boldsymbol{t}_{\alpha}$ can be interpreted as the surface-intrinsic part of $\boldsymbol{v}$.

\section{Entropy production}

The set of equations of dynamics derived in the previous section, Eq. (49), Eq. (50) and Eq. (51), involve both the currents describing the transport processes in the tangent space of the dividing surface, $j_{x}^{\alpha}$, and the currents describing the transverse transport processes, which in turn involve the boundary values of the bulk hydrodynamic fields. In order that the equations form a closed set, relations between the currents and the surface thermodynamic/hydrodynamic fields should be developed from the equation of entropy balance, according to one of the basic principles of non-equilibrium thermodynamics. In this section, we present the derivation of the equation of entropy balance from which entropy production associated with transport processes is identified [24].

The tangential currents can be decomposed into two parts, a reactive part, which is associated with reversible transport processes, and a dissipative part, which is associated with irreversible processes:

$$
\begin{aligned}
& j_{A}^{\alpha}=j_{A, \mathrm{r}}^{\alpha}+j_{A, \mathrm{~d}}^{\alpha} \\
& j_{p}^{\alpha}=j_{p, \mathrm{r}}^{\alpha}+j_{p, \mathrm{~d}}^{\alpha}, \\
& j_{e}^{\alpha}=j_{e, \mathrm{r}}^{\alpha}+j_{e, \mathrm{~d}}^{\alpha},
\end{aligned}
$$

\footnotetext{
4 This is due to the fact that, when the dividing surface is curved, the volume measure and the area measure will then have a different dependence on the distance to the dividing surface.
}

where the subscript " $\mathrm{r}$ " denotes the reactive part and " $\mathrm{d}$ " the dissipative. Once an expression for the entropy production is obtained, the reactive parts of the currents can be determined, by the definition that they should not contribute to the entropy production; moreover, and more importantly, thermodynamic/hydrodynamic "forces" driving the dissipative currents can also be identified [2526].

The equation of entropy balance in its general form can be written as

$$
D_{t} s=-D_{\alpha} j_{s}^{\alpha}+j_{s}^{-}-j_{s}^{+}+\sigma_{s}
$$

where $j_{s}^{\alpha}$ represents the tangential current of entropy, $j_{s}^{ \pm}$ are transverse currents, and $\sigma_{s}$ is the density of entropy production. The derivation of an explicit expression of the equation starts from calculating, based on Eq. (24), the variations with time of all the thermodynamic quantities and isolating $D_{t} s$ as

$$
\begin{aligned}
D_{t} s=\frac{1}{T}\left[D_{t} e\right. & +\boldsymbol{f}_{\mathrm{rs}} \cdot \partial_{t} \boldsymbol{R}-\boldsymbol{v} \cdot D_{t} \boldsymbol{p} \\
& \left.-\sum_{A} \mu^{A} D_{t} n_{A}-D_{\alpha}\left(\boldsymbol{S}^{\alpha} \cdot \partial_{t} \boldsymbol{R}\right)\right] .
\end{aligned}
$$

Further derivation can be carried out by using the conservation laws, Eq. (49) to Eq. (51), to replace the corresponding time derivatives and using the identity derived in Section 3.2, Eq. (38), to replace $\boldsymbol{f}_{\mathrm{rs}} \cdot \partial_{t} \boldsymbol{R}$ with

$$
\begin{aligned}
& \boldsymbol{f}_{\mathrm{rs}} \cdot \partial_{t} \boldsymbol{R}=\boldsymbol{f}_{\mathrm{rs}} \cdot\left(\boldsymbol{v}-v^{\alpha} \boldsymbol{t}_{\alpha}\right) \\
& =\boldsymbol{f}_{\mathrm{rs}} \cdot \boldsymbol{v}+v^{\alpha}\left(\boldsymbol{p} \cdot \partial_{\alpha} \boldsymbol{v}+s \partial_{\alpha} T+\sum_{A} n_{A} \partial_{\alpha} \mu^{A}\right)
\end{aligned}
$$

where Eq. (58) has been used. With the use of $\boldsymbol{f}_{\mathrm{rs}}=$ $D_{\alpha} \boldsymbol{S}_{(0)}^{\alpha}$ in addition, the equation of entropy balance can be rearranged into

$$
\begin{aligned}
D_{t} s=- & D_{\alpha}\left[\frac { 1 } { T } \left(j_{e}^{\alpha}+\boldsymbol{S}^{\alpha} \cdot \partial_{t} \boldsymbol{R}-\sum_{A} \mu^{A} j_{A}^{\alpha}\right.\right. \\
& \left.\left.-\boldsymbol{v} \cdot \boldsymbol{j}_{p}^{\alpha}-\boldsymbol{v} \cdot \boldsymbol{S}_{(0)}^{\alpha}\right)\right]+j_{s}^{-}-j_{s}^{+} \\
+ & {\left[\left(j_{e}^{\alpha}+\boldsymbol{S}^{\alpha} \cdot \partial_{t} \boldsymbol{R}-\sum_{A} \mu^{A} j_{A}^{\alpha}-\boldsymbol{v} \cdot \boldsymbol{j}_{p}^{\alpha}\right.\right.} \\
& -\frac{1}{T}\left(\boldsymbol{j}_{p}^{\alpha}+\boldsymbol{S}_{(0)}^{\alpha}-\boldsymbol{p} v^{\alpha}\right) \cdot \partial_{\alpha} \boldsymbol{v} \\
- & \left.\frac{1}{T} \sum_{A}\left(j_{A}^{\alpha}-n_{A} v^{\alpha}\right) \partial_{\alpha} \mu^{A}-\frac{1}{T} \sum_{K} \xi^{K} \Gamma_{K}\right] \\
+ & {\left[\frac{1}{T}\left(j_{e}^{-}-j_{e}^{+}\right)-\frac{1}{T} \boldsymbol{v} \cdot\left(\boldsymbol{j}_{p}^{-}-\boldsymbol{j}_{p}^{+}\right)\right.} \\
& \left.-\frac{1}{T} \sum_{A} \mu^{A}\left(j_{A}^{-}-j_{A}^{+}\right)-\left(j_{s}^{-}-j_{s}^{+}\right)\right]
\end{aligned}
$$


where $\Gamma_{K} \equiv \sum_{A} \mu^{A} \nu_{A, K}$ is the affinity for reaction $K$. The interpretations of the different terms in the equation are made clear immediately by a comparison with the general form, Eq. (62). The terms collected in the first pair of square brackets give the tangential current of entropy,

$$
\begin{gathered}
j_{s}^{\alpha}=\frac{1}{T}\left(j_{e}^{\alpha}+\boldsymbol{S}^{\alpha} \cdot \partial_{t} \boldsymbol{R}-\sum_{A} \mu^{A} j_{A}^{\alpha}-\boldsymbol{v} \cdot \boldsymbol{j}_{p}^{\alpha}\right. \\
\left.-\boldsymbol{v} \cdot \boldsymbol{S}_{(0)}^{\alpha}\right) .
\end{gathered}
$$

The terms in the second and third pairs of square brackets sum up the entropy production from all of the membranerelated transport processes. Those in the second pair represent the entropy production from transport processes intrinsic to the dividing surface, and those in the third describe the entropy production from processes of transport between the bulk fluids and the dividing surface.

\subsection{The intrinsic transport processes}

The reactive parts of the currents can now be determined from the explicit expression of the entropy production given in Eq. (65). An examination of the terms enclosed by the second pair of square brackets yields

$$
\begin{gathered}
\boldsymbol{j}_{p, \mathrm{r}}^{\alpha}=-\boldsymbol{S}_{(0)}^{\alpha}+\boldsymbol{p} v^{\alpha}, \\
j_{A, \mathrm{r}}^{\alpha}=n_{A} v^{\alpha}
\end{gathered}
$$

and

$$
j_{e, \mathrm{r}}^{\alpha}=v^{\alpha}\left(\boldsymbol{v} \cdot \boldsymbol{p}+T s+\sum_{A} \mu^{A} n_{A}\right)-\boldsymbol{S}^{\alpha} \cdot \partial_{t} \boldsymbol{R}
$$

because these currents alone, in the absence of the dissipative parts, make no contribution to the entropy production. Finally, it can be concluded that the reactive and dissipative parts of $j_{s}^{\alpha}$ defined in Eq.(66) are given, respectively, by

$$
\begin{aligned}
& j_{s, \mathrm{r}}^{\alpha}=s v^{\alpha} \\
& j_{s, \mathrm{~d}}^{\alpha}=\frac{1}{T}\left(j_{e, \mathrm{~d}}^{\alpha}-\boldsymbol{v} \cdot \boldsymbol{j}_{p, \mathrm{~d}}^{\alpha}-\sum_{A} \mu^{A} j_{A, \mathrm{~d}}^{\alpha}\right) .
\end{aligned}
$$

In the most general sense, the above identifications of the reactive currents are not complete. We will discuss this point again where constitutive relations are derived.

The various dissipative currents, $\boldsymbol{j}_{p, \mathrm{~d}}^{\alpha}, j_{A, \mathrm{~d}}^{\alpha}$ 's, and $j_{e, \mathrm{~d}}^{\alpha}$ can now be determined. However, not all of the $j_{A, \mathrm{~d}}^{\alpha}$ 's are independent due to a constraint

$$
\sum_{A} m^{A} j_{A, \mathrm{~d}}^{\alpha}=0
$$

which follows from Eq. (56) and Eq. (68). Thus, $j_{A, \mathrm{~d}}^{\alpha}$ of any species $A$ can be chosen to be the one dependent on the rest. Given that a judicious choice $A=O$ can be made for one reason or another, Eq. (72) can be written as

$$
j_{O, \mathrm{~d}}^{\alpha}=-\sum_{A \neq O} \frac{m^{A}}{m^{O}} j_{A, \mathrm{~d}}^{\alpha} .
$$

The entropy production from the intrinsic dissipative processes can finally be expressed in terms of the various independent dissipative currents, $j_{p, \mathrm{~d}}^{\alpha}, j_{A \neq O, \mathrm{~d}}^{\alpha}$ 's, and $j_{e, \mathrm{~d}}^{\alpha}$ and the thermodynamic forces driving them:

$$
\begin{aligned}
T \sigma_{s, \|}= & -j_{s, \mathrm{~d}}^{\alpha} \partial_{\alpha} T-\boldsymbol{j}_{p, \mathrm{~d}}^{\alpha} \cdot \partial_{\alpha} \boldsymbol{v}-\sum_{A \neq O} j_{A, \mathrm{~d}}^{\alpha} \partial_{\alpha} \tilde{\mu}^{A} \\
& -\sum_{K} \xi^{K} \Gamma_{K}
\end{aligned}
$$

where

$$
\tilde{\mu}^{A} \equiv \mu^{A}-\frac{m^{A}}{m^{O}} \mu^{O}=\left.\frac{1}{\sqrt{g}} \frac{\delta E}{\delta n_{A}}\right|_{\boldsymbol{p}, \rho, s, \boldsymbol{R},\left\{n_{B \neq A, O}\right\}} .
$$

\subsection{The processes of transport between the surface and the bulk fluids}

The processes of transport between the surface and the bulk fluids contribute to the total entropy production in the form of those terms contained in the last pair of square brackets in Eq. (65). It is clear that the contributions depend not only on the boundary behaviour of the bulk hydrodynamic fields, but also on the surface hydrodynamic fields. A more illuminating expression of the contributions can be derived as follows.

The values of the bulk currents evaluated at the dividing surface appear in Eq. (48) which defines the transverse currents, $j_{e}^{ \pm}, j_{p}^{ \pm}, j_{A}^{ \pm}$'s and $j_{s}^{ \pm}$, and they are given, respectively, by

$$
\boldsymbol{J}_{e}^{ \pm}=\left(e^{ \pm}+p^{ \pm}\right) \boldsymbol{v}_{ \pm}-\mathrm{T}_{\mathrm{d}}^{ \pm} \cdot \boldsymbol{v}_{ \pm}+\boldsymbol{J}_{q}^{ \pm}
$$

where $\mathrm{T}_{\mathrm{d}}^{ \pm}$is the viscous stress of the corresponding bulk fluid and $\boldsymbol{J}_{q}^{ \pm}$is the heat flux;

$$
\mathrm{J}_{p}^{ \pm}=\rho^{ \pm} \boldsymbol{v}_{ \pm} \boldsymbol{v}_{ \pm}+p^{ \pm} \mathbf{I}-\mathrm{T}_{\mathrm{d}}^{ \pm},
$$

where I is the identity tensor;

$$
\boldsymbol{J}_{A}^{ \pm}=n_{A}^{ \pm} \boldsymbol{v}_{ \pm}+\boldsymbol{J}_{A, \mathrm{~d}}^{ \pm}
$$

and

$$
\boldsymbol{J}_{s, \mathrm{~d}}^{ \pm}=\left(\boldsymbol{J}_{q}^{ \pm}-\mu_{ \pm}^{A} \boldsymbol{J}_{A, \mathrm{~d}}^{ \pm}\right) / T_{ \pm} .
$$

Inserting these explicit expressions into Eq. (48) yields

$$
\begin{aligned}
\boldsymbol{j}_{p}^{ \pm}= & \rho^{ \pm}\left[\boldsymbol{n} \cdot\left(\boldsymbol{v}_{ \pm}-\boldsymbol{v}\right) \boldsymbol{v}_{ \pm}\right]+p^{ \pm} \boldsymbol{n}-\boldsymbol{n} \cdot \mathrm{T}_{\mathrm{d}}^{ \pm}, \\
j_{e}^{ \pm}= & \rho^{ \pm} \boldsymbol{v}_{ \pm}^{2}\left[\boldsymbol{n} \cdot\left(\boldsymbol{v}_{ \pm}-\boldsymbol{v}\right)\right]+p^{ \pm} \boldsymbol{n} \cdot \boldsymbol{v} \\
& -\boldsymbol{n} \cdot \mathrm{T}_{\mathrm{d}}^{ \pm} \cdot \boldsymbol{v}_{ \pm}+\sum_{A} \mu_{ \pm}^{A} j_{A}^{ \pm}+T_{ \pm} j_{s}^{ \pm},
\end{aligned}
$$


where $\boldsymbol{n} \cdot \boldsymbol{v}=\boldsymbol{n} \cdot \partial_{t} \boldsymbol{R}$ has been used.

The sum of the entropy production from all the processes of transport between the surface and the bulk fluids can now be expressed as

$$
\begin{aligned}
T \sigma_{s, \perp} \equiv & j_{e}^{-}-j_{e}^{+}-\boldsymbol{v} \cdot\left(j_{p}^{-}-j_{p}^{+}\right) \\
& -\sum_{A} \mu^{A}\left(j_{A}^{-}-j_{A}^{+}\right)-T\left(j_{s}^{-}-j_{s}^{+}\right) \\
= & \sum_{A}\left[j_{A}^{-}\left(\mu_{-}^{A}-\mu^{A}\right)-j_{A}^{+}\left(\mu_{+}^{A}-\mu^{A}\right)\right] \\
& +j_{s}^{-}\left(T_{-}-T\right)-j_{s}^{+}\left(T_{+}-T\right) \\
& +\left(-\boldsymbol{n} \cdot \mathrm{T}_{\mathrm{d}}^{-} \cdot \boldsymbol{n}+\frac{1}{2} \rho^{-}\left(\boldsymbol{v}_{-}-\boldsymbol{v}\right)^{2}\right. \\
& \left.+\frac{1}{2} \rho^{-} \boldsymbol{v}_{-}^{2}-\frac{1}{2} \rho^{-} \boldsymbol{v}^{2}\right) \boldsymbol{n} \cdot\left(\boldsymbol{v}_{-}-\boldsymbol{v}\right) \\
& +\left(\boldsymbol{n} \cdot \mathrm{T}_{\mathrm{d}}^{+} \cdot \boldsymbol{n}-\frac{1}{2} \rho^{+}\left(\boldsymbol{v}_{+}-\boldsymbol{v}\right)^{2}\right. \\
& \left.\quad-\frac{1}{2} \rho^{+} \boldsymbol{v}_{+}^{2}+\frac{1}{2} \rho^{+} \boldsymbol{v}^{2}\right) \boldsymbol{n} \cdot\left(\boldsymbol{v}_{+}-\boldsymbol{v}\right) \\
& -\left(\boldsymbol{n} \cdot \mathrm{T}_{\mathrm{d}}^{-} \cdot \boldsymbol{t}_{\alpha}\right)\left[\boldsymbol{t}^{\alpha} \cdot\left(\boldsymbol{v}_{-}-\boldsymbol{v}\right)\right] \\
& +\left(\boldsymbol{n} \cdot \mathrm{T}_{\mathrm{d}}^{+} \cdot \boldsymbol{t}_{\alpha}\right)\left[\boldsymbol{t}^{\alpha} \cdot\left(\boldsymbol{v}_{+}-\boldsymbol{v}\right)\right]
\end{aligned}
$$

In the above expression, the currents $j_{A}^{ \pm}$'s and $\boldsymbol{n} \cdot\left(\boldsymbol{v}_{ \pm}-\boldsymbol{v}\right)$ are related by

$$
\sum_{A} m^{A} j_{A}^{ \pm}=\rho^{ \pm} \boldsymbol{n} \cdot\left(\boldsymbol{v}_{ \pm}-\boldsymbol{v}\right)
$$

which follows from $\sum_{A} m^{A} \boldsymbol{J}_{A}^{ \pm}=\rho^{ \pm} \boldsymbol{v}^{ \pm}$, Eq. (48) and Eq. (57). Consequently, $j_{A}^{ \pm}$'s for all $A$ values may be chosen as the independent currents, or alternatively, $\rho^{ \pm} \boldsymbol{n} \cdot\left(\boldsymbol{v}_{ \pm}-\boldsymbol{v}\right)$ and $\left\{j_{A}^{ \pm}, A \neq 0^{ \pm}\right\}$may be used as the independent currents, where $0^{+}$and $0^{-}$denote the two judiciously chosen species, whose currents will be eliminated explicitly. gives

Making the latter choice and rewriting Eq. (82) finally

$$
\begin{aligned}
T \sigma_{s, \perp}= & j_{s}^{-}\left(T_{-}-T\right)-j_{s}^{+}\left(T_{+}-T\right) \\
& +\sum_{A \neq 0^{ \pm}} j_{A}^{-} \Delta \mu_{-}^{A}-\sum_{A \neq 0^{ \pm}} j_{A}^{+} \Delta \mu_{+}^{A} \\
& +\left(-\boldsymbol{n} \cdot \mathrm{T}_{\mathrm{d}}^{-} \cdot \boldsymbol{n}+\Pi_{-}\right) \boldsymbol{n} \cdot\left(\boldsymbol{v}_{-}-\boldsymbol{v}\right) \\
& +\left(\boldsymbol{n} \cdot \mathrm{T}_{\mathrm{d}}^{+} \cdot \boldsymbol{n}-\Pi_{+}\right) \boldsymbol{n} \cdot\left(\boldsymbol{v}_{+}-\boldsymbol{v}\right) \\
& -\left(\boldsymbol{n} \cdot \mathrm{T}_{\mathrm{d}}^{-} \cdot \boldsymbol{t}_{\alpha}\right)\left[\boldsymbol{t}^{\alpha} \cdot\left(\boldsymbol{v}_{-}-\boldsymbol{v}\right)\right] \\
& +\left(\boldsymbol{n} \cdot \mathrm{T}_{\mathrm{d}}^{+} \cdot \boldsymbol{t}_{\alpha}\right)\left[\boldsymbol{t}^{\alpha} \cdot\left(\boldsymbol{v}_{+}-\boldsymbol{v}\right)\right] .
\end{aligned}
$$

The newly-introduced quantities, $\Delta \mu_{ \pm}^{A}$ and $\Pi_{ \pm}$, are defined by

$$
\begin{aligned}
\Delta \mu_{ \pm}^{A} \equiv & \left(\mu_{ \pm}^{A}-\frac{m^{A}}{m^{0^{ \pm}}} \mu_{ \pm}^{0^{ \pm}}\right) \\
& -\left(\mu^{A}-\frac{m^{A}}{m^{0^{ \pm}}} \mu^{0^{ \pm}}\right), \quad A \neq 0^{ \pm}, \\
\Pi_{ \pm} \equiv & \frac{\rho^{ \pm}}{m^{0^{ \pm}}}\left[\frac{1}{2} m^{0^{ \pm}}\left(\boldsymbol{v}_{ \pm}-\boldsymbol{v}\right)^{2}+\left(\mu_{ \pm}^{0^{ \pm}}+\frac{1}{2} m^{0^{ \pm}} \boldsymbol{v}_{ \pm}^{2}\right)\right. \\
& \left.-\left(\mu^{0^{ \pm}}+\frac{1}{2} m^{0^{ \pm}} \boldsymbol{v}^{2}\right)\right] .
\end{aligned}
$$

Their physical interpretations become more evident when it is recalled that $\left(\mu_{ \pm}^{A}+\frac{1}{2} m^{A} \boldsymbol{v}_{ \pm}^{2}\right)$ and $\left(\mu^{A}+\frac{1}{2} m^{A} \boldsymbol{v}^{2}\right)$ are, respectively, the bulk and surface chemical potentials of species $A$ in the corresponding rest frames.

\section{Constitutive relations}

The expressions of the entropy production derived in the previous Section allow us to identify the conjugate "force" current pairs associated with all the different dissipative processes. In this Section, we describe how physically meaningful relations between the currents and the forces are developed, under the assumption that the non-equilibrium dynamics of the system is within the linear regime. To follow the standard terminology of statistical physics, we will call those relations constitutive relations in general 272829 .

\subsection{Symmetry based classification of forces and currents}

Eq. (74) and Eq. (84) involve many different currents, such as $j_{s, \mathrm{~d}}^{\alpha}, \boldsymbol{j}_{p, \mathrm{~d}}^{\alpha}, j_{A, \mathrm{~d}}^{\alpha}$ 's, etc., and many different driving forces, such as $\partial_{\alpha} T, \partial_{\alpha} \boldsymbol{v}, \partial_{\alpha} \tilde{\mu}^{A}$, etc. ${ }^{5}$ It is well known that a single current may be driven by several different forces. A systematic way to identify all the possible different forces driving a particular current is the following: first, to classify all the forces and currents according to their behaviour under a group of orthogonal transformations, consisting of both rotations of the internal coordinate system and the inversion of the local normal vector to the dividing surface; and then, to determine whether the symmetry of the system allows for, or forbids, a force of one type, generically represented by $F^{i}$, to drive a current of another type, denoted $J_{j}$. Here, the Roman superscripts/subscripts label such classifications of forces and currents.

The generic classes of behaviour of a quantity under the coordinate transformations consist of the following:

\footnotetext{
${ }^{5}$ In the formalistic sense what quantities are called forces and what are called currents are completely arbitrary. The convention we have adopted here conforms with either physical intuition or historical usages.
} 
scalar, vector, and tensors of different ranks, which transform like a scalar, vector, and tensor with respect to internal coordinate transformations, but which remain invariant with respect to an inversion of $\boldsymbol{n}$; pseudo-scalar, pseudo-vector, and pseudo-tensors, which are different from scalar, vector, and tensors only in that they change their sign under the inversion of $\boldsymbol{n}$.

For the intrinsic dissipative processes involved in Eq. (74), the classification with respect to internal coordinate transformations is more essential. When applied this leads to the following conclusions:

a) Genuine chemical reactions involve forces $F^{K}$ and currents $J_{K}$, which are given, respectively, by

$$
F^{K}=-\Gamma_{K}=-\sum_{A} \mu^{A} \nu_{A, K}, \quad J_{K}=\xi^{K} .
$$

Whether $F^{K}$ and $J_{K}$ are scalars or pseudoscalars depends on the precise nature of a reaction. However, when a chemical reaction process $K^{*}$ refers to the "flipflop" from one side of the membrane to the other of molecules of a particular chemical species, as described by Eq. (52), the corresponding force and current

$$
F^{K^{*}}=-\left(\mu^{\bar{C}^{+}}-\mu^{\bar{C}^{-}}\right), \quad J_{K^{*}}=\xi^{K^{*}},
$$

are clearly pseudoscalars.

b) Heat conduction and diffusion in the surface involve forces, $F_{\alpha}^{s}$ and $\left\{F_{\alpha}^{A}, A \neq O\right\}$, and currents, $j_{s, \mathrm{~d}}^{\alpha}$ and $\left\{j_{A, \mathrm{~d}}^{\alpha}, A \neq O\right\}$, which are

$$
\begin{array}{ll}
F_{\alpha}^{s} \equiv-\partial_{\alpha} T, & F_{\alpha}^{A} \equiv-\partial_{\alpha} \tilde{\mu}^{A}, \\
J_{s}^{\alpha} \equiv j_{s, \mathrm{~d}}^{\alpha}, & J_{A}^{\alpha} \equiv j_{A, \mathrm{~d}}^{\alpha} .
\end{array}
$$

These forces and currents transform like vectors under the rotations of the internal coordinate system.

c) The dissipative momentum transport involves forces and currents, whose representations need both a tensor and a vector,

$$
\begin{aligned}
\partial_{\alpha} \boldsymbol{v} & =\left(\partial_{\alpha} \boldsymbol{v} \cdot \boldsymbol{t}_{\beta}\right) \boldsymbol{t}^{\beta}+\left(\partial_{\alpha} \boldsymbol{v} \cdot \boldsymbol{n}\right) \boldsymbol{n} \\
& \equiv-\left(F_{\alpha \beta} \boldsymbol{t}^{\beta}+F_{(n), \alpha} \boldsymbol{n}\right) \\
\boldsymbol{j}_{p, \mathrm{~d}}^{\alpha} & =\left(\boldsymbol{j}_{p, \mathrm{~d}}^{\alpha} \cdot \boldsymbol{t}^{\beta}\right) \boldsymbol{t}_{\beta}+\left(\boldsymbol{j}_{p, \mathrm{~d}}^{\alpha} \cdot \boldsymbol{n}\right) \boldsymbol{n} \\
& \equiv J_{p}^{\alpha \beta} \boldsymbol{t}_{\beta}+J_{p,(n)}^{\alpha} \boldsymbol{n} .
\end{aligned}
$$

Thus, $F_{(n), \alpha}$ and $J_{p,(n)}^{\alpha}$ form the (pseudo)vector forcecurrent pair. The tensorial part, $F_{\alpha \beta}$, is not a symmetric tensor in general and can be decomposed into three contributions,

$$
\begin{aligned}
F_{\alpha \beta}=\frac{1}{2}[ & g_{\alpha \beta} F_{\gamma}^{\gamma}+\left(F_{\alpha \beta}+F_{\beta \alpha}-g_{\alpha \beta} F_{\gamma}^{\gamma}\right) \\
& \left.+\varepsilon_{\alpha \beta} \varepsilon^{\gamma \delta} F_{\gamma \delta}\right]
\end{aligned}
$$

each of which is invariant under any internal-coordinate transformation and each of which should be considered as an independent force. The corresponding currents in $J_{\alpha \beta}$ can be identified in a similar way.
For the transport processes that give rise to the entropy production given in Eq. (84), it is more meaningful to use linear combinations of the apparent forces and currents to generate new forces and currents that either remain invariant or change sign under an inversion of $\boldsymbol{n}$. Before we discuss the new forces and currents, a change of notation will be made concerning our reference to species. So far, the definition of the labelled species, represented by $A$, is used to keep the derivation concise. In what follows, the chemical identities of the molecular species will be explicitly referred to, in order that the physical interpretations of quantities associated with material transport become more apparent. Specifically, $C$ will be used to represent those molecular species, which have been assumed to reach chemical equilibrium across the membrane quasistatically, while $\bar{C}$ will denote those molecular species which are assumed not to permeate the membrane quasistatically. With this notation and with the choice that the " 0 " in the species expression $0^{ \pm}$used in Eq. (84) refers to one of the $\bar{C}$ species, the relevant terms in Eq. (84) acquire an alternative expression

$$
\begin{aligned}
& \sum_{A \neq 0^{ \pm}} j_{A}^{-} \Delta \mu_{-}^{A}-\sum_{A \neq 0^{ \pm}} j_{A}^{+} \Delta \mu_{+}^{A} \\
& =\sum_{C}\left(j_{C}^{-} \Delta \mu_{-}^{C}-j_{C}^{+} \Delta \mu_{+}^{C}\right) \\
& \quad+\sum_{\bar{C} \neq 0}\left(j_{\bar{C}^{-}}^{-} \Delta \mu_{-}^{\bar{C}^{-}}-j_{\bar{C}^{+}}^{+} \Delta \mu_{+}^{\bar{C}^{+}}\right) .
\end{aligned}
$$

From Eq. (84) the new forces and currents can now be derived for the various different transport processes, as follows.

a) The first four lines of the equation sum up the contributions from the absorption and conduction of heat by the surface as well as from the adsorption onto, and permeation across, the surface of the constituent molecules. Genuine scalar forces $F_{(\mathrm{s})}$ and currents $J^{(\mathrm{s})}$, as well as pseudoscalar forces $F_{(\text {a) }}$ and currents $J^{(\text {a) }}$, are given by

$$
\begin{aligned}
& F_{(\mathrm{s})}^{s}=-\left(T^{+}+T^{-}-2 T\right), \\
& J_{s}^{(\mathrm{s})}=\frac{1}{2}\left(j_{s}^{+}-j_{s}^{-}\right), \\
& F_{(\mathrm{a})}^{s}=-\left(T^{+}-T^{-}\right), \\
& J_{s}^{(\mathrm{a})}=\frac{1}{2}\left(j_{s}^{+}+j_{s}^{-}\right),
\end{aligned}
$$

$$
\begin{aligned}
& F_{(\mathrm{s})}^{C}=-\left(\Delta \mu_{+}^{C}+\Delta \mu_{-}^{C}\right), \\
& J_{C}^{(\mathrm{s})}=\frac{1}{2}\left(j_{C}^{+}-j_{C}^{-}\right), \\
& F_{(\mathrm{a})}^{C}=-\left(\Delta \mu_{+}^{C}-\Delta \mu_{-}^{C}\right), \\
& J_{C}^{(\mathrm{a})}=\frac{1}{2}\left(j_{C}^{+}+j_{C}^{-}\right),
\end{aligned}
$$




$$
\begin{gathered}
F_{(\mathrm{s})}^{\bar{C}}=-\left(\Delta \mu_{+}^{\bar{C}^{+}}+\Delta \mu_{-}^{\bar{C}^{-}}\right), \\
J_{\bar{C}}^{(\mathrm{s})}=\frac{1}{2}\left(j_{\bar{C}^{+}}^{+}-j_{\bar{C}^{-}}^{-}\right), \\
F_{(\mathrm{a})}^{\bar{C}}=-\left(\Delta \mu_{+}^{\bar{C}^{+}}-\Delta \mu_{-}^{\bar{C}^{-}}\right), \\
J_{\bar{C}}^{(\mathrm{a})}=\frac{1}{2}\left(j_{\bar{C}^{+}}^{+}+j_{\bar{C}^{-}}^{-}\right), \\
F_{(\mathrm{s})}^{\rho}=\frac{1}{2}\left[\boldsymbol{n} \cdot\left(\mathrm{T}_{\mathrm{d}}^{+}+\mathrm{T}_{\mathrm{d}}^{-}\right) \cdot \boldsymbol{n}-\Pi_{+}-\Pi_{-}\right], \\
J_{\rho}^{(\mathrm{s})}=\boldsymbol{n} \cdot\left(\boldsymbol{v}_{+}-\boldsymbol{v}_{-}\right), \\
F_{(\mathrm{a})}^{\rho}=\frac{1}{2}\left[\boldsymbol{n} \cdot\left(\mathrm{T}_{\mathrm{d}}^{+}-\mathrm{T}_{\mathrm{d}}^{-}\right) \cdot \boldsymbol{n}-\Pi_{+}+\Pi_{-}\right], \\
J_{\rho}^{(\mathrm{a})}=\boldsymbol{n} \cdot\left(\boldsymbol{v}_{+}+\boldsymbol{v}_{-}-2 \boldsymbol{v}\right) .
\end{gathered}
$$

The interpretations of the scalar currents and the pseudoscalar currents are rather obvious: The scalar currents describe the heat absorbed, the material adsorbed, and the pseudoscalar ones describe heat conduction, material permeation across the surface.

b) The last two lines of Eq. (84) describe the dissipation associated with the hydrodynamic "slip" between the surface and the two contacting bulk fluids. The forces and currents involved can be written as genuine vectors and pseudovectors,

$$
\begin{aligned}
F_{(\mathrm{s}), \alpha}^{p} & =\boldsymbol{t}_{\alpha} \cdot\left(\boldsymbol{v}_{+}+\boldsymbol{v}_{-}-2 \boldsymbol{v}\right), \\
J_{p}^{(\mathrm{s}), \alpha} & =\frac{1}{2} \boldsymbol{n} \cdot\left(\mathrm{T}_{\mathrm{d}}^{+}-\mathrm{T}_{\mathrm{d}}^{-}\right) \cdot \boldsymbol{t}^{\alpha}, \\
F_{(\mathrm{a}), \alpha}^{p} & =\boldsymbol{t}_{\alpha} \cdot\left(\boldsymbol{v}_{+}-\boldsymbol{v}_{-}\right), \\
J_{p}^{(\mathrm{a}), \alpha} & =\frac{1}{2} \boldsymbol{n} \cdot\left(\mathrm{T}_{\mathrm{d}}^{+}+\mathrm{T}_{\mathrm{d}}^{-}\right) \cdot \boldsymbol{t}^{\alpha} .
\end{aligned}
$$

\subsection{Constitutive relations}

The total entropy production associated with the dividing surface, the sum of Eq. (74) and Eq. (84), can now be expressed in terms of the forces and currents defined above:

$$
T \sigma_{s} \equiv T \sigma_{s, \|}+T \sigma_{s, \perp}=\sum_{i} J_{i} F^{i},
$$

where $i$ runs over all the classes listed above. The basis for developing constitutive relations is our requirement that $T \sigma_{s}$ be positive definite, although it is developed for the dividing surface in the Gibbs model system, which is not a physical surface. This requirement ensures the thermodynamic stability of any equilibrium state described by the theory; its validity will be discussed in the last section of the paper.

The most general form of the constitutive relations is given by $27 / 2829]$

$$
J_{i}=\sum_{j} \Omega_{i j} F^{j},
$$

where $\Omega_{i j}$ are phenomenological parameters. It immediately follows from Onsager's reciprocal relations that $\Omega_{i j}$ 's must satisfy a general property: $\Omega_{i j}=\Omega_{j i}$ if $J_{i}$ and $J_{j}$ have the same parity under time reversal, and $\Omega_{i j}=-\Omega_{j i}$ if $J_{i}$ and $J_{j}$ have the opposite parity. The positive definitiveness of $T \sigma_{s}$ is then ensured if matrix $\Omega_{i j}$ is positive definite. The anti-symmetric elements of $\Omega_{i j}$ require a few more words. It is not difficult to see that they do not contribute to the entropy production, in other words, any possible couplings characterized by anti-symmetric $\Omega_{i j}$ are in fact reactive couplings rather than dissipative couplings.

In principle, any of the currents, $J_{i}$, may be driven by any of the forces, $F_{j}$, leading to the so-called crosscoupling. In other words, in the absence of pertinent symmetries or invariances, all forms of cross coupling are possible. However, if the physical description of the system is invariant with respect to some or all of the orthogonal transformations, then the invariance will eliminate certain cross-couplings. For example, if the equilibrium shape of the membrane (or the dividing surface) is symmetric with respect to rotations around any local normal vector, then scalar, vector and tensor forces can only drive currents of the same type, leaving the coefficients of all the related cross-coupling terms zero. Similarly, if the physics of the system does not distinguish one side of the membrane from the other, a pseudoscalar force can not drive a scalar current and a scalar force can not a pseudoscalar current. Consequently, the corresponding $\Omega_{i j}$ 's vanish. If it turns out that these symmetries in practice are not exact, but almost correct, then the corresponding cross-couplings will be weak. An obvious approximation is to discard those cross-coupling terms. It may be worth noting, however, that symmetry properties alone are not sufficient for identifying physically meaningful and relevant forces, currents, and constitutive relations. The general physics embodied in the entropy-production equations and the specific physics of a system under consideration are necessarily needed.

A few explicitly written constitutive relations may lead to some more intuitive understanding of the general discussion given above. For example, concerning the surfaceintrinsic processes described by Eq. (89), there are

$$
\begin{aligned}
& J_{s}^{\alpha}=K_{T}\left(-\partial^{\alpha} T\right)+\sum_{A \neq O} \Omega_{s, A}\left(-\partial^{\alpha} \tilde{\mu}^{A}\right), \\
& J_{A}^{\alpha}=\sum_{B \neq O} \Omega_{A, B}\left(-\partial^{\alpha} \tilde{\mu}^{B}\right)+\Omega_{A, s}\left(-\partial^{\alpha} T\right) .
\end{aligned}
$$

These two relations should be familiar to the reader, where $K_{T}$ may be interpreted as the effective heat conductivity of the surface, and $\Omega_{A, B}$ 's are the effective dissipation coefficients associated with diffusion. Another example can be developed based on Eq. (90) and Eq. (91), and has the following form: is the following:

$$
\begin{aligned}
\boldsymbol{j}_{p, \mathrm{~d}}^{\alpha}= & -\eta_{\mathrm{s}}\left(g^{\alpha \gamma} g^{\beta \delta}+g^{\alpha \delta} g^{\beta \gamma}-g^{\alpha \beta} g^{\gamma \delta}\right)\left(\partial_{\gamma} \boldsymbol{v} \cdot \boldsymbol{t}_{\delta}\right) \boldsymbol{t}_{\beta} \\
& -\zeta_{\mathrm{s}}\left(\partial_{\beta} \boldsymbol{v} \cdot \boldsymbol{t}^{\beta}\right) \boldsymbol{t}^{\alpha}
\end{aligned}
$$

where the phenomenological constants $\zeta_{\mathrm{s}}$ and $\eta_{\mathrm{s}}$ may be interpreted as the only relevant surface viscosity coefficients. This constitutive relation is a two-dimensional analogue of the familiar expression of viscous stress in a bulk 
fluid. To be sure, the symmetry reasoning alone would allow for the presence in the above constitutive relation of both the last antisymmetric part of the force in Eq. (91) and a "force" term of the form $\left(\partial^{\alpha} \boldsymbol{v} \cdot \boldsymbol{n}\right) \boldsymbol{n}$. The physical requirement that no dissipation should be associated with uniform rotational motion of the whole system, however, renders those two terms absent. The latter term was allowed to be present in the work reported in Ref. 30, though.

The processes of transport of material across the surface are also of practical interest. One of such processes is the so-called flip-flop process. In the real world of membrane systems it often occurs that this process is so slow as to be practically irrelevant for experimental observations 18. However, situations where this process is relevant are also encountered 31, where the kinetic rate characterizing the process can be determined experimentally. In the framework of our theory, the simplest form of the constitutive relation associated with the flip-flop of a chemical species $\bar{C}$ is given by

$$
\xi^{K^{*}}=\Omega_{\uparrow}\left[-\left(\mu^{\bar{C}^{+}}-\mu^{\bar{C}^{-}}\right)\right],
$$

where the phenomenological parameter $\Omega_{\uparrow}$ may be related to experimentally determined flip-flop rate. A related constitutive relation concerns the permeation of molecules of a $\bar{C}$ type. As it has been pointed out in connection with Eq. (98), the current of permeation, $J_{\bar{C}}^{(\text {a) }}$, is a pseudoscalar, thus can be driven by $F_{(\text {a) }}^{\bar{C}}$ as well as by $F^{K^{*}}=$ $-\left(\mu^{\bar{C}^{+}}-\mu^{\bar{C}^{-}}\right)$defined in Eq. (88). The corresponding constitutive relation may be written as

$$
\begin{aligned}
J_{\bar{C}^{(a)}}= & \Omega_{\bar{C}, \text { perm }}^{(1)}\left[-\left(\Delta \mu_{+}^{\bar{C}^{+}}-\Delta \mu_{-}^{\bar{C}^{-}}\right)\right] \\
& +\Omega_{\bar{C}, \text { perm }}^{(2)}\left[-\left(\mu^{\bar{C}^{+}}-\mu^{\bar{C}^{-}}\right)\right] .
\end{aligned}
$$

In contrast, in the constitutive relation characterizing the permeation of molecules of a $C$-type, only the counterpart of the dissipative coefficient $\Omega_{\bar{C} \text {,perm }}^{(1)}$ exists.

The final example concerns the total permeation of material across the surface described by the forces and currents defined in Eq. (100). If it may be assumed that there is no cross coupling between the scalar and the pseudoscalar quantities, then the following simple constitutive relation may be written:

$$
\begin{aligned}
\boldsymbol{n} \cdot & \left(\boldsymbol{v}_{+}+\boldsymbol{v}_{-}-2 \boldsymbol{v}\right) \\
& =\Omega_{\mathrm{perm}} \frac{1}{2}\left[\boldsymbol{n} \cdot\left(\mathrm{T}_{\mathrm{d}}^{+}-\mathrm{T}_{\mathrm{d}}^{-}\right) \cdot \boldsymbol{n}-\Pi_{+}+\Pi_{-}\right] .
\end{aligned}
$$

The constant $\Omega_{\text {perm }}$ is related to membrane permeability. This relation would be the familiar Darcy's law 32, but for the presence of the viscous stresses from the bulk fluids.

\subsection{From constitutive relations to equations of motion}

In the above, we have presented a framework of principles for developing constitutive relations, the specific forms of which must depend on the particular physical system under consideration. We end this whole section with some comments on how the general theory may be considered from an operational point of view.

The complete set of equations of motion may be divided into two subsets: those that describe the hydrodynamics of the bulk "filler" fluids, i.e., Eq. (43), and those that describe the dynamics of the surface excess fields, i.e., Eq. (44). Or, more specifically, Eq. (49) to Eq. (51), together with the one that governs the conformational dynamics, Eq. (57), or Eq. (58). These two subsets of equations can only be solved if specific constitutive relations are given or developed. The constitutive relations associated with transverse transport processes provide the necessary coupling between the boundary values of the bulk hydrodynamic fields and the surface dynamic fields, as Eq. (109) illustrates, for example. They may be viewed and used as boundary conditions for the bulk equations of motion. An operational strategy may then be to solve first the bulk equations of motion and express all the boundary quantities of the bulk fluids appearing in the surface equations of motion in terms of the surface dynamic fields, and then obtain an effective, closed set of equations of motion for the surface dynamic fields. As the focus of a study of dynamics of a membrane is naturally on the surface fields, the effective equations should be the basis for analyzing the dynamics of the membrane.

The number of the final, effective equations of motion, or the number of relevant dynamic fields, associated with the dividing surface can be worked out in a general fashion. Similar to the case of a bulk fluid, relevant conserved variables are given by the surface densities of excess energy, excess momentum, and numbers of excess particles, represented by $e, \boldsymbol{p}$ and $\left\{n_{A}\right\}$, respectively, as in Eq. (49) to Eq. (51). An extra, non-conserved, and scalar, dynamic variable, however, becomes necessary for describing the conformational aspect of membrane dynamics, as mentioned in the preceding paragraph ${ }^{6}$. For a reader with a particular interest in the linear analysis of equations of dynamics, the total number of the relevant dynamic fields in principle determines the number of independent dynamic modes that are implied by the effective equations of motion. In practice, the number and the specific nature of those modes that are relevant to, or observable in, experimental observations of the dynamics of a particular system will have to be determined on a case-dependent basis.

Given its importance and accessibility for experimental observations [2] the conformational aspect of membrane dynamics on its own merits a comment. Eq. (57) may be considered the corresponding equation of motion, and clearly, it is coupled to the other equations of motion. That coupling is expected to be complicated in a general case. In some limit cases, however, an explicit form of the

${ }^{6}$ In principle, the shape field, $\boldsymbol{R}$ is a vector, implying three scalar variables. But, only one variable, corresponding to the motion of the membrane in its normal direction, is the physically relevant one, as a consequence of the reparametrization invariance [30]. 
equation is available. One of such limit cases, the case of membranes made of single-component lipid-bilayers, has been explored earlier 7 and will also be discussed later in Sec. 7.2 of this paper. An effective equation for the conformational dynamics in that limit case can be found in [7.

\section{The general theory in a few limit cases}

The formulation of the theory as presented in the previous sections is kept very general. This feature is useful, seen from the formalistic point of view. A few limit cases are, however, interesting from the practical point of view. In fact, another prediction of our theory, which is in principle non-trivial, will be brought out more clearly in one of the limit cases. In this section we will discuss these limit cases.

\subsection{Multi-component membrane with no-slip boundary conditions}

In treatment of the bulk hydrodynamics of a viscous fluid in contact with a boundary, the boundary condition that is usually imposed is the so-called no-slip boundary condition, where the bulk fluid at the boundary is constrained to have the velocity of the boundary material.

If this limit may be assumed as a valid one for the membrane-fluid system under our consideration, its implementation does not appear straightforward, due to the peculiar structural characteristics of the membrane. On the one hand, one type of molecular building blocks of the membrane are amphiphilic molecules, which are distributed in two opposing monolayers, each in contact with a different bulk fluid. There is no physical reason to assume that the lipid molecules forming the two different monolayers should have the same collective velocities, and in turn, no reason to assume that the boundary velocities of the two bulk fluids are the same. On the other hand, another type of the molecular building blocks are transmembrane proteins, which span the whole thickness of the membrane and which contribute to the dynamic interaction between the membrane and both of the bulk fluids. The question is, in other words, what surface velocities should be matched tangentially with the boundary velocities of the two contacting bulk fluids. The previously defined surface velocity $\boldsymbol{v}$, which is the center-of-mass velocity of the whole surface, obviously is not the appropriate one.

We propose, as an answer to the question, that the noslip boundary conditions be implemented by matching the boundary velocities of the two bulk fluids with two in principle different linear combinations of the velocities of all the different species associated with the dividing surface. By use of the fact that the collective velocity of a particular species $A$ is given by $n_{A} \boldsymbol{v}_{A} \equiv \boldsymbol{j}_{A}=\left(j_{A, \mathrm{~d}}^{\alpha} \boldsymbol{t}_{\alpha}+n_{A} \boldsymbol{v}\right)$, the no-slip boundary conditions can be formulated as

$$
\boldsymbol{v}_{ \pm}=\sum_{A} L_{ \pm}^{A}\left(j_{A, \mathrm{~d}}^{\alpha} \boldsymbol{t}_{\alpha}+n_{A} \boldsymbol{v}\right)
$$

The $L_{ \pm}^{A}$ 's in the expression are phenomenological quantities, and may be interpreted as the effective areas of the different species "seen" by the bulk fluids. Note that $\sum_{A} L_{ \pm}^{A} n_{A}=1$ must be satisfied in order that the expression also hold for the case of uniform motion of all species.

Using this constraint together with Eq. (72) leads to an alternative expression of the no-slip boundary conditions

$$
\boldsymbol{v}_{ \pm}=\boldsymbol{v}+\sum_{A \neq O} \tilde{L}_{ \pm}^{A} j_{A, \mathrm{~d}}^{\alpha} \boldsymbol{t}_{\alpha}
$$

where

$$
\tilde{L}_{ \pm}^{A} \equiv L_{ \pm}^{A}-\frac{m^{A}}{m^{O}} L_{ \pm}^{O} .
$$

Eq. (111) implies that $\boldsymbol{t}^{\alpha} \cdot\left(\boldsymbol{v}_{+}-\boldsymbol{v}\right)$ and $\boldsymbol{t}^{\alpha} \cdot\left(\boldsymbol{v}_{-}-\boldsymbol{v}\right)$ are no longer independent of the currents $\left\{j_{A, \mathrm{~d}}^{\alpha}, A \neq O\right\}$, and it can then be used to eliminate $\boldsymbol{t}^{\alpha} \cdot\left(\boldsymbol{v}_{ \pm}-\boldsymbol{v}\right)$ from those terms in Eq. (84) that contain them. Collecting all the terms in the total entropy production which contain $\left\{j_{A, \mathrm{~d}}^{\alpha}, A \neq O\right\}$, including those in Eq. (74), finally leads to the identification of

$$
F_{\alpha}^{A}=-\partial_{\alpha} \tilde{\mu}^{A}+\left(\tilde{L}_{+}^{A} \boldsymbol{n} \cdot \mathrm{T}_{\mathrm{d}}^{+} \cdot \boldsymbol{t}_{\alpha}-\tilde{L}_{-}^{A} \boldsymbol{n} \cdot \mathrm{T}_{\mathrm{d}}^{-} \cdot \boldsymbol{t}_{\alpha}\right)
$$

as the thermodynamic force conjugate to the diffusion current $J_{A}^{\alpha}$.

The terms contained in parenthesis in the above equation represent contributions from the two bulk fluids, and they underscore one of the specific predictions of our theory: the exchange of momentum between the surface and the bulk fluids is coupled to the dissipative processes in the surface. At the level of principles, this is a non-trivial prediction, and is made, to our knowledge, for the first time here. In Section 8$]$ an order-of-magnitude estimate of this new effect will be made with a view towards its experimental verification.

\subsection{Single-component lipid bilayers}

The case where the membrane is a bilayer composed of a single species of lipid molecules is also worth discussing in connection with the earlier works dealing with such a system [67]. In those earlier works, the description of the hydrodynamics of the membrane was established in an intuition-based manner rather than from a systematic derivation. In this section, we will demonstrate that the earlier description can be formulated as a limit case of the formalism developed here, thus putting the earlier description on a firmer basis.

In the earlier description, a number of limiting assumptions were made. First, the temperature was assumed to be uniform in the whole system; secondly, the membrane was assumed to be impermeable to solution (water) molecules; thirdly, it was assumed that no transport of the lipid molecules took place either between the bulk fluids and the membrane or between the two distinct monolayers of the membrane; fourthly, it was assumed that the intramonolayer shear dissipations could be neglected, but that the 
dissipation associated with the relative motion between the two monolayers was relevant; finally, the Stokes condition was assumed, i.e. that the inertia of the membrane surface could also be neglected. Consistent with these assumptions, the single-component lipid bilayer was considered as a composite of two systems $(+/-)$, one for each monolayer. Consequently, the description of the dynamics became much simplified, consisting of two continuity equations

$$
D_{t} n_{l_{ \pm}}+D_{\alpha} j_{l_{ \pm}}^{\alpha} \equiv D_{t} n_{l_{ \pm}}+D_{\alpha}\left(n_{l_{ \pm}} W_{ \pm}^{\alpha}\right)=0
$$

where $\boldsymbol{v}_{l_{ \pm}}=W_{ \pm}^{\alpha} \boldsymbol{t}_{\alpha}+\partial_{t} \boldsymbol{R}$ were the velocities of the lipid molecules in the two monolayers, and by two additional equations which correspond to the conservations of the monolayer momenta under the Stokes condition,

$$
\boldsymbol{f}_{\mathrm{rs}}^{ \pm} \pm \mathrm{T}^{ \pm} \cdot \boldsymbol{n}+\boldsymbol{f}_{\mathrm{m}}^{ \pm}=0
$$

$\boldsymbol{f}_{\mathrm{rs}}^{ \pm}$represented the restoring force acting on each $(+/-)$ monolayer, which should be derived from the thermodynamic free energy, and $\boldsymbol{f}_{\mathrm{m}}^{ \pm}$was the force acting on one monolayer by the other. As a model for the intermonolayer dissipation, the following phenomenological expression was employed

$$
\boldsymbol{t}^{\alpha} \cdot \boldsymbol{f}_{\mathrm{m}}^{ \pm}=-b \boldsymbol{t}^{\alpha} \cdot\left(\boldsymbol{v}_{l_{ \pm}}-\boldsymbol{v}_{l_{\mp}}\right)=-b\left(W_{ \pm}^{\alpha}-W_{\mp}^{\alpha}\right),
$$

where $b$ was the "intermonolayer friction coefficient." In order that the equations of the dynamics be completely closed, intuition-based expressions were proposed for $\boldsymbol{f}_{\mathrm{rs}}^{ \pm}$, which satisfied

$$
\boldsymbol{f}_{\mathrm{rs}}^{ \pm} \cdot \boldsymbol{t}_{\alpha}=-n_{l_{ \pm}} \partial_{\alpha} \mu^{l_{ \pm}}
$$

and

$$
\boldsymbol{f}_{\mathrm{rs}}^{+}+\boldsymbol{f}_{\mathrm{rs}}^{-}=\boldsymbol{f}_{\mathrm{rs}}
$$

Eq. (115) can now be expressed in an alternative form, which will make a comparison with the current formalism easier. The sum of the two subequations yields the equation corresponding to the momentum conservation for the whole bilayer, which is given by

$$
\boldsymbol{f}_{\mathrm{rs}}+\mathrm{T}^{+} \cdot \boldsymbol{n}-\mathrm{T}^{-} \cdot \boldsymbol{n}=0 ;
$$

and the subtraction of the two subequations leads to the following expression

$$
\begin{aligned}
\frac{1}{2}\left(j_{l_{+}, \mathrm{d}}^{\alpha}-j_{l_{-}, \mathrm{d}}^{\alpha}\right) & \\
=\left(\frac{n_{l_{+}} n_{l_{-}}}{n_{l_{+}}+n_{l_{-}}}\right)^{2} \frac{1}{b} & {\left[\boldsymbol{n} \cdot\left(\frac{1}{n_{l_{+}}} \mathrm{T}^{+}-\frac{1}{n_{l_{-}}} \mathbf{T}^{-}\right) \cdot \boldsymbol{t}^{\alpha}\right.} \\
& \left.-\partial^{\alpha}\left(\mu^{l_{+}}-\partial^{\alpha} \mu^{l_{-}}\right)\right]
\end{aligned}
$$

where

$$
j_{l_{ \pm}, \mathrm{d}}^{\alpha} \equiv j_{l_{ \pm}}^{\alpha}-n_{l_{ \pm}} \frac{j_{\rho}^{\alpha}}{\rho}=j_{l_{ \pm}}^{\alpha}-n_{l_{ \pm}} \frac{j_{l_{+}}^{\alpha}+j_{l_{-}}^{\alpha}}{n_{l_{+}}+n_{l_{-}}},
$$

obviously satisfying $j_{l_{+}, \mathrm{d}}^{\alpha}=-j_{l_{-}, \mathrm{d}}^{\alpha}$.

When the same system is considered within the current formalism under the same limiting conditions, the equations of dynamics for the system have the same formal expressions as Eq. (114) and Eq. (119), where $j_{l_{ \pm}, \mathrm{d}}^{\alpha}$ defined by Eq. (121) represent the "diffusion currents." If, in addition to all the limiting conditions already mentioned, the limiting, no-slip boundary conditions Eq. (111) are also used, which in this simple case reduce to

$$
\boldsymbol{v}_{+}=\boldsymbol{v}_{l_{+}}, \quad \boldsymbol{v}_{-}=\boldsymbol{v}_{l_{-}}
$$

or equivalently to

$$
\tilde{L}_{+}^{l_{+}}=L_{+}^{l_{+}}=\frac{1}{n^{l_{+}}}, \quad \tilde{L}_{-}^{l_{+}}=-L_{-}^{l_{-}}=-\frac{1}{n^{l_{-}}},
$$

the constitutive relation concerning the "diffusion currents" is given by, following Eq. (113),

$$
\begin{aligned}
j_{l_{+}, \mathrm{d}}^{\alpha}=-j_{l_{-}, \mathrm{d}}^{\alpha}=\frac{1}{2}\left(j_{l_{+}, \mathrm{d}}^{\alpha}-j_{l_{-}, \mathrm{d}}^{\alpha}\right) \\
=\Omega_{\mathrm{D}}\left[\boldsymbol{n} \cdot\left(\frac{1}{n_{l_{+}}} \mathrm{T}_{\mathrm{d}}^{+}-\frac{1}{n_{l_{-}}} \mathrm{\top}_{\mathrm{d}}^{-}\right) \cdot \boldsymbol{t}^{\alpha}\right. \\
\left.\quad-\partial_{\alpha}\left(\mu^{l_{+}}-\mu^{l_{-}}\right)\right]
\end{aligned}
$$

It is obvious that, if the following identification is made,

$$
\Omega_{\mathrm{D}}=\left(\frac{n_{l_{+}} n_{l_{-}}}{n_{l_{+}}+n_{l_{-}}}\right)^{2} \frac{1}{b},
$$

the above constitutive relation becomes equivalent to Eq. (120). The above equation makes it clear that the term "diffusion current" within our formalism has a broader meaning than its canonical one, in that it describes relative motion between species and does not have to be associated with processes of molecular mixing.

\subsection{Incompressible membrane and bulk fluids}

Theoretical work dealing with a generic membrane system often in practice employs the assumption that both the bulk fluids surrounding the membrane and the membrane itself are incompressible. This assumption is justified in most cases. With a view of applications of our general theory to those practical situations, we in this subsection discuss the limit case of volumewise incompressible fluids and membrane.

In what follows, we will model the limiting condition of incompressibility by assuming that the average molecular volume associated with each molecular species in the membrane-fluid system remains constant throughout the system, in other words, being insensitive to the spatial inhomogeneities in other thermodynamic variables. In addition we will only consider the case where another limiting condition holds, that there is no transport of any molecules across the membrane. This latter condition implies, according to the definition of "species" established 
earlier, that in the Gibbs-model description of the system, two labelled species, $\bar{C}^{+}$and $\bar{C}^{-}$, are associated with a single chemical species $\bar{C}$. It is not difficult to see that these conditions amount to two separate constraints on the surface fields $\left\{n_{\bar{C}^{ \pm}}\right\}$. For our purpose, the two constraints are expressed in the following form

$$
n_{0^{ \pm}}=B_{ \pm}\left(\left\{n_{\bar{C}^{+} \neq 0^{+}}, n_{\bar{C}^{-} \neq 0^{-}}\right\}\right),
$$

where $\bar{C}=0$ represents the molecular species (water) forming the solvent.

The immediate consequence of the constraints is that, among all the transverse currents of material transport, $\left(\left\{j_{\bar{C}^{ \pm} \neq 0^{ \pm}}^{ \pm}\right\}, \rho^{ \pm} \boldsymbol{n} \cdot\left(\boldsymbol{v}_{ \pm}-\boldsymbol{v}\right)\right)$, two - one for the “+"-region and one for the "-"-region - must become dependent on the rest as well as on the surface currents, $\left\{j_{\bar{C}^{ \pm}, \mathrm{d}}^{\alpha}\right\}$. There are different ways to remove these dependent currents from the entropy-production equation. An obvious one is by direct substitutions of their explicit dependences on the other currents. Although straightforward, the implementation is tedious and changes the formulistic structure of the derivations we have described so far. As a formulistically much more concise and simpler alternative, we have developed a different approach to implement the constraints, which is sketched below.

In our approach, the following energy-density function is introduced first

$$
\begin{aligned}
& e\left(n_{0^{+}}, n_{0^{-}},\left\{n_{\bar{C}^{+} \neq 0^{+}}, n_{\bar{C}^{-} \neq 0^{-}}\right\}\right) \\
& =e_{\text {phys }}\left(\left\{n_{\bar{C}^{+} \neq 0^{+}}, n_{\bar{C}^{-} \neq 0^{-}}\right\}\right) \\
& \quad+\lambda^{0^{+}}\left(n_{0^{+}}-B_{+}\right)+\lambda^{0^{-}}\left(n_{0^{-}}-B_{-}\right),
\end{aligned}
$$

where $e_{\text {phys }}\left(\left\{n_{\bar{C}^{+} \neq 0^{+}}, n_{\bar{C}^{-} \neq 0^{-}}\right\}\right)$represents the physical energy-density function for the constrained system, and where $\lambda^{0^{ \pm}}$are two multipliers to be determined. It is obvious that, when the constraints are used, $e\left(n_{0^{+}}, n_{0^{-}}\right.$, $\left\{n_{\bar{C}^{+} \neq 0^{+}}, n_{\bar{C}^{-} \neq 0^{-}}\right\}$), coincides with the physical function $e_{\text {phys }}\left(\left\{n_{\bar{C}^{+} \neq 0^{+}}, n_{\bar{C}^{+} \neq 0^{+}}\right\}\right)$no matter what values the two multipliers take, and this fact will be taken advantage of in what follows.

In the derivation of the entropy production the system is then treated formally as if it were not constrained by letting $e\left(n_{0^{+}}, n_{0^{-}},\left\{n_{\bar{C}^{+} \neq 0^{+}}, n_{\bar{C}^{+} \neq 0^{+}}\right\}\right)$play the role of the surrogate of the physical energy-density function. The final expression of the entropy production,

$$
\begin{aligned}
T \sigma_{s}= & -j_{s, \mathrm{~d}}^{\alpha} \partial_{\alpha} T-\boldsymbol{j}_{p, \mathrm{~d}}^{\alpha} \cdot \partial_{\alpha} \boldsymbol{v}-\sum_{A \neq O} j_{A, \mathrm{~d}}^{\alpha} \partial_{\alpha} \tilde{\mu}^{A} \\
& -\sum_{K} \xi^{K} \Gamma_{K}+j_{s}^{-}\left(T_{-}-T\right)-j_{s}^{+}\left(T_{+}-T\right) \\
& +\sum_{\bar{C} \neq 0}\left(j_{\bar{C}^{-}}^{-} \Delta \mu_{-}^{\bar{C}^{-}}-j_{\bar{C}^{+}}^{+} \Delta \mu_{+}^{\bar{C}^{+}}\right) \\
& +\left(-\boldsymbol{n} \cdot \mathrm{T}_{\mathrm{d}}^{-} \cdot \boldsymbol{n}+\Pi_{-}\right) \boldsymbol{n} \cdot\left(\boldsymbol{v}_{-}-\boldsymbol{v}\right) \\
& +\left(\boldsymbol{n} \cdot \mathrm{T}_{\mathrm{d}}^{+} \cdot \boldsymbol{n}-\Pi_{+}\right) \boldsymbol{n} \cdot\left(\boldsymbol{v}_{+}-\boldsymbol{v}\right) \\
& -\left(\boldsymbol{n} \cdot \mathrm{T}_{\mathrm{d}}^{-} \cdot \boldsymbol{t}_{\alpha}\right)\left[\boldsymbol{t}^{\alpha} \cdot\left(\boldsymbol{v}_{-}-\boldsymbol{v}\right)\right] \\
& +\left(\boldsymbol{n} \cdot \mathrm{T}_{\mathrm{d}}^{+} \cdot \boldsymbol{t}_{\alpha}\right)\left[\boldsymbol{t}^{\alpha} \cdot\left(\boldsymbol{v}_{+}-\boldsymbol{v}\right)\right]
\end{aligned}
$$

thus looks formulistically identical to the sum of Eq. (74) and Eq. (84), except that the multipliers $\lambda^{0^{ \pm}}$appear in the places of the surface chemical potentials $\mu^{0^{ \pm}}$in

$$
\begin{aligned}
& \Delta \mu_{ \pm}^{\bar{C}^{ \pm}} \equiv\left(\mu_{ \pm}^{\bar{C}^{ \pm}}-\frac{m^{\bar{C}}}{m^{0}} \mu_{ \pm}^{0^{ \pm}}\right) \\
&-\left(\mu^{\bar{C}^{ \pm}}-\frac{m^{\bar{C}}}{m^{0}} \lambda^{0^{ \pm}}\right), \quad \bar{C} \neq 0, \\
& \Pi_{ \pm} \equiv \frac{\rho^{ \pm}}{m^{0}}\left[\frac{1}{2} m^{0}\left(\boldsymbol{v}_{ \pm}-\boldsymbol{v}\right)^{2}+\left(\mu_{ \pm}^{0^{ \pm}}+\frac{1}{2} m^{0} \boldsymbol{v}_{ \pm}^{2}\right)\right. \\
&\left.-\left(\lambda^{0^{ \pm}}+\frac{1}{2} m^{0} \boldsymbol{v}^{2}\right)\right]
\end{aligned}
$$

Eq. (128) still contains all currents, both independent ones and the corresponding dependent ones. The freedom in choosing the values of the multipliers $\lambda^{0^{ \pm}}$when the constraints are satisfied now means that, given a particular choice of the independent currents, the corresponding dependent ones can then be eliminated from Eq. (128) by choosing values for $\lambda^{0^{ \pm}}$accordingly. For example, for the choice where $\left(\left\{j_{\bar{C}^{ \pm}}^{ \pm}, \bar{C} \neq 0\right\}\right)$ are used as the independent transverse currents, the values of $\lambda^{0^{ \pm}}$must be chosen such that

$$
\boldsymbol{n} \cdot \mathrm{T}_{\mathrm{d}}^{ \pm} \cdot \boldsymbol{n}-\Pi_{ \pm}=0 .
$$

This choice renders both the fourth and the fifth lines in Eq. (128) zero, thus eliminating the corresponding dependent currents $\rho^{ \pm} \boldsymbol{n} \cdot\left(\boldsymbol{v}_{ \pm}-\boldsymbol{v}\right)$.

The values of $\lambda^{0^{ \pm}}$chosen as such, together with the constitutive relations which can be derived consequently, should then be used in conjunction with equations of motion where the constraints are explicitly satisfied, in order that the whole formulation of the dynamics form a selfconsistent and closed one.

The implications of Eq. (131) merit a short comment. It follows from the the definition of $\Pi_{ \pm}$that the values of $\lambda^{0^{ \pm}}$are linearly related to the chemical potentials of the bulk solvent molecules, which in turn are related to the pressures in the bulk fluids. Thus, bulk-pressure gradients tangential to the surface, $\boldsymbol{t}_{\alpha} \cdot \boldsymbol{\nabla} p_{ \pm}$, are translated by Eq. (131) into tangential gradients of $\lambda^{0^{ \pm}}$and can thereby drive diffusion currents within the surface in principle, as implied by Eq. (113). The practical relevance of this mechanism can, however, be argued to be insignificant. A quick estimate reveals that the tangential gradients of $\lambda^{0^{ \pm}}$are given roughly by $w^{0} \boldsymbol{t}_{\alpha} \cdot \nabla p_{ \pm}$, where $w^{0}$ denotes the molecular volume of the solvent. When compared with the contributions in Eq. (113) from the bulk shear stresses, this effect is suppressed likely by the ratio between a molecular length and the wavelength characterizing the bulkpressure gradients, thus probably insignificant. This suppression means that, although the excess quantity of the solvent molecules associated with the surface may not be negligible, its effect on the motion of other surface-related molecular species may be assumed to be insignificant. Our 
understanding here justifies, therefore, the use of this assumption in the previous works on membrane hydrodynamics [ 7.10.

A general remark may also be made to conclude this subsection. Although our method of constraint implementation has been applied in the specific limit case of systems consisting of incompressible fluids and incompressible membranes, it may also be applied to cases where constraints are different. The difference in the use of the method will only result from the different details of the constraint functions used in Eq. (126).

\section{Discussions}

In the previous sections, a formulation of hydrodynamics of the membrane-fluid systems has been presented in a very general form, both in terms of equations of motion based on the basic conservation laws and in terms of derived constitutive relations. Although an application of the general theory to any specific system is beyond the scope of this paper, we will, nevertheless, discuss in this section a number of general issues which must be dealt with in applications and which will also make the connection between the theory and experimental situations visible. For the following discussion we reiterate that the theory is only an effective one and that its purpose is to give a mesoscopic/macroscopic-scale description of the membrane dynamics, which can be connected to experimental descriptions on the corresponding scales.

The first issue concerns the structure and the content of the theory. From the way the theory is constructed, it is clear that its description of the motion in the regions close to the membrane interface is, by design, not correct qualitatively. The obvious questions are, then, how the quality of the theory can be controlled, and what criteria should be used as measures of the quality of the theory. The means of the control is already contained in the theory itself and rests on tuning the set of kinetic coefficients associated with the various constitutive relations. Whenever the theory should be applied, it is assumed that the hydrodynamic conditions at the boundaries of the entire system are controlled by macroscopic means and are, therefore, known. Given those boundary conditions, and provided that the dynamical behaviour of the system in the regions sufficiently far away from the membrane (or the dividing surface), or the "bulk behaviour," is known already, or may be known, by "measurements," the set of kinetic coefficients can then be tuned in the process of solving the equations of motion under the given boundary conditions, such that the quantitative description of the "bulk behaviour" given by the theory matches that given by the measurements to some desired degree of agreement. Moreover, in the theory, a few quantities pertaining to the interfacial region can be calculated,

1. $\int_{\bar{\Sigma}} d V \bar{x}(\boldsymbol{r}, t)$,

2. $\int_{\bar{B}_{ \pm}^{\alpha}} d \overline{\boldsymbol{A}} \cdot\left[\overline{\boldsymbol{J}}_{X}-\bar{x} \partial_{t}(\boldsymbol{R}+h \boldsymbol{n})\right]$, where $\bar{\Sigma}$ is the familiar volume element already used in Section 3.1 and

$$
\begin{gathered}
\bar{B}_{ \pm}^{\alpha}=\left\{\boldsymbol{R}\left(\bar{\xi}^{1}, \bar{\xi}^{2}, t\right)+h \boldsymbol{n}\left(\bar{\xi}^{1}, \bar{\xi}^{2}, t\right) \mid\right. \\
\xi^{3-\alpha}-\Delta \xi^{3-\alpha} / 2 \leq \bar{\xi}^{3-\alpha} \leq \xi^{3-\alpha}+\Delta \xi^{3-\alpha} / 2 \\
\left.-\epsilon^{-} \leq h \leq \epsilon^{+}, \quad \bar{\xi}^{\alpha}=\xi^{\alpha} \pm \Delta \xi^{\alpha} / 2\right\}
\end{gathered}
$$

represent the side surfaces of $\bar{\Sigma}$ and $d \overline{\boldsymbol{A}}$ is the area element on this surface times its outward-pointing normal vector. The counterparts of these quantities, or at least some of them, in the real physical system may also be obtained from measurements. One example would be the measurements of distribution and motion of membrane proteins within the membrane by the use of modern techniques such as Fluorescence Confocal Microscopy [33] and Fluorescence Correlation Spectroscopy [34. Such experimental information on the "surface behaviour" of the system, whenever available, must also be used to tune the theoretical kinetic coefficients, thereby to control the quality of the theory.

Besides tuning the kinetic coefficients such that the quantities labelled 1 . and 2. above are identical for the Gibbs model and the experiment, the membrane parameters should also be tuned such that the position of the shape $\boldsymbol{R}$ matches the position of the dividing surface in the experiment.

The second issue is a general one concerning the tuning of the kinetic coefficients. It has been a requirement, made in Section 6.2, that the entropy production in the theory be positive definite in order that the equilibrium states of the theory be stable. One consequence of this requirement, among others, is that kinetic coefficient $\Omega_{i i}$ for each $i$ is positive. It turns out that in certain situations, this stability requirement can only be fulfilled by an approximation in the tuning of the kinetic coefficients. The following example of permeation of molecules across a membrane illustrates the point.

Consider the following simplified picture of a real (in contrast to the Gibbs model) membrane-fluid system. The membrane is taken to be a homogeneous slab of material of thickness $2 d$. A certain molecular species has different diffusion constants, $D^{\prime}$ and $D$, in the membrane region and in the two bulk fluids, respectively. At a distance $L_{1}$ from the membrane slab, there is a reservoir of the molecules with a constant chemical potential $\mu_{1}$, and similarly on the other side, a reservoir of chemical potential $\mu_{2}<\mu_{1}$ is placed at a distance $L_{2}$ from the membrane slab.

The quantity of interest is the steady-state molecular flux, $j^{\text {real }}$, between the two reservoirs. It is easy to work out that

$$
j^{\text {real }}=\frac{D D^{\prime}}{D^{\prime}\left(L_{1}+L_{2}\right)+2 d D}\left(\mu_{1}-\mu_{2}\right) .
$$

In the theory, the real system is replaced by a Gibbs model system: two bulk fluids, where the molecular species has diffusion constant $D$, separated by an infinitely thin dividing interface with an effective kinetic coefficient $\Omega$ for 
permeation of the molecules, which must be tuned. The two reservoirs of chemical potentials $\mu_{1}$ and $\mu_{2}$ are then at distances $L_{1}+d$ and $L_{2}+d$ from the interface. In order that the steady-state molecular flux be reproduced in the model system, it is necessary that the boundary chemical potentials of the bulk fluids at the interface be different, denoted by $\mu^{+}$and $\mu^{-}$. The steady-state flux, $j^{\text {model }}$, can then be expressed as

$$
\begin{aligned}
j^{\text {model }} & =-D \frac{\mu_{2}-\mu^{+}}{L_{2}+d}=-\Omega\left(\mu^{+}-\mu^{-}\right) \\
& =-D \frac{\mu^{-}-\mu_{1}}{L_{1}+d} .
\end{aligned}
$$

From this equation an alternative expression of $j^{\text {model }}$ can be obtained

$$
j^{\text {model }}=\frac{D \Omega}{\Omega\left(L_{1}+L_{2}+2 d\right)+D}\left(\mu_{1}-\mu_{2}\right) .
$$

In order that the requirement, $j^{\text {model }}=j^{\text {real }}$, be fulfilled, $\Omega$ must be set to be

$$
\Omega=\frac{D D^{\prime}}{2 d\left(D-D^{\prime}\right)} .
$$

Eq. (136) immediately illustrates a problem of the theory in the case where the molecules diffuse faster than they do in the bulk fluids, i.e. $D^{\prime}>D$ : on the one hand, the effective kinetic coefficient $\Omega$ must be given a negative value; on the other hand, any equilibrium state becomes unstable in the theory when $\Omega$ is negative.

This problem does not, however, render the theory inapplicable to the case. A reasonable approximation can still be made, which involves setting $\Omega$ to infinity. The chemical boundary condition for the bulk fluids at the dividing surface thus becomes $\mu^{+}=\mu^{-}$. The error introduced in this approximation has an upper bound, which can be easily calculated:

$$
\begin{aligned}
\frac{j^{\text {real }}-j^{\text {model }}}{j^{\text {real }}} & =\frac{2 d}{L_{1}+L_{2}+2 d} \cdot \frac{D^{\prime}-D}{D^{\prime}} \\
& <\frac{2 d}{L_{1}+L_{2}} .
\end{aligned}
$$

Clearly, the theory should still give quantitatively reasonable result if $d$ is much smaller than $L_{1}$ or $L_{2}$, or in a more general situation, if the length scale associated with the chemical gradient in the system is much larger than the microscopic thickness of the membrane ${ }^{7}$.

\footnotetext{
7 The negative permeability is a direct consequence of invoking the Gibbs model in cases where $D^{\prime}>D$. An alternative formulation may also be envisioned, as suggested by one of the referees of this manuscript. In this formulation, the thickness of the membrane would be taken to zero without corresponding "compensation" in the theory for the effects that are associated with the finite thickness of the membrane. As a consequence, the kinetic coefficient associated with transmembrane permeation stays positive in all cases. This zero-thickness treatment is obviously an approximation, as is our suggested solution to the problem of negative permeability. The errors introduced due to this approximation may be comparable to those associated with our formulation.
}

The last issue to be touched upon here is the question of how the various kinetic coefficients in the effective theory may be related to experimental data on the corresponding transport processes. The precise answer to the question depends on the nature of the experiments that are done. For example, if a permeation experiment is done in the way described above, where the molecular flux is measured, then an effective kinetic coefficient $\Omega$ can be derived based on Eq. 135. Unfortunately, very few experiments have been done so far on the investigations of membrane-involved transport processes; concrete situations where this issue can be addressed specifically are hard to find. We hope, however, that our theory may provide some guidelines for designing useful experiments regarding membrane-involved transport processes and for interpreting data obtained.

It would add strength to a general theory such as the one presented in this paper if it makes conceptually non-trivial and experimentally relevant predictions of new physical effects. Indeed, our theory does make predictions that are conceptually non-trivial, among which the following constitutive relation - the simplest one that can be written down based on Eq. (113),

$$
\begin{aligned}
j_{A, \mathrm{~d}}^{\alpha}= & \Omega_{A, A} g^{\alpha \beta} F_{\beta}^{A}=\Omega_{A, A} g^{\alpha \beta}\left[-\partial_{\beta} \tilde{\mu}^{A}\right. \\
& \left.+\left(\tilde{L}_{+}^{A} \boldsymbol{n} \cdot \mathbf{T}_{\mathrm{d}}^{+} \cdot \boldsymbol{t}_{\beta}-\tilde{L}_{-}^{A} \boldsymbol{n} \cdot \mathbf{T}_{\mathrm{d}}^{-} \cdot \boldsymbol{t}_{\beta}\right)\right],
\end{aligned}
$$

is the most notable. An order-of-magnitude estimate of the effect predicted by Eq. (138), namely, that the viscous stresses exerted by the bulk fluids on the membrane can drive the surface diffusion processes in the membrane, may shed some light on the experimental relevance of this theoretical prediction.

Consider the case where a membrane is composed of a lipid species and a protein species embedded in the lipid bilayer. The subject of our interest is the motion of the embedded protein. Assume that the physical conditions are such that there exists a surface gradient of the protein concentration in the membrane, sufficient to drive appreciable protein diffusion. In addition, the membrane is subjected to a shear flow induced in one or both of the bulk fluids. For an order-of-magnitude estimate it suffices that we assume near-plane geometry for the membrane and that we include in the chemical potential of the protein only the contribution from entropy of mixing, i.e.

$$
\mu^{\mathrm{p}}=k T \ln n_{\mathrm{p}} .
$$

It follows immediately then that

$$
\partial_{\alpha} \mu^{\mathrm{p}} \sim \frac{k T}{n_{\mathrm{p}}} \frac{\Delta n_{\mathrm{p}}}{\Delta l}
$$

where $\Delta l$ denotes the wavelength characterizing the concentration gradient. The contribution from the bulk shear stress can also be estimated, based on Eq. (138), to be

$$
\tilde{L}^{\mathrm{p}} \cdot\left(\eta \frac{\Delta v}{\Delta l}\right)
$$


where $\tilde{L}^{\mathrm{p}}$ is in order of magnitude similar to $\tilde{L}_{ \pm}^{\mathrm{p}}$ defined by Eq. (112) with the species index $O$ referring to the lipid, but in explicit expression different from $\tilde{L}_{ \pm}^{\mathrm{p}}[19$.

For the newly predicted effect to be comparable to the effect associated with the chemical gradient, the variation of the velocity characterizing the bulk shear flow should be

$$
\Delta v \sim \frac{k T}{\eta \tilde{L}^{\mathrm{p}}} \frac{\Delta n_{\mathrm{p}}}{n_{\mathrm{p}}} .
$$

Taking $\eta_{0}=10^{-3} \mathrm{~Pa} \cdot \mathrm{s}$, the shear viscosity of water at room temperature $\left(k T=4 \cdot 10^{-21} \mathrm{~J}\right)$ and setting $\tilde{L}^{\mathrm{p}} \sim$ $500 \AA^{2}=5 \cdot 10^{-18} \mathrm{~m}^{2}$, a reasonable value for the crosssectional area of a typical transmembrane-protein molecule, we get

$$
\Delta v \sim\left(\frac{\Delta n_{\mathrm{p}}}{n_{\mathrm{p}}} \cdot \frac{\eta_{0}}{\eta}\right) \frac{\mathrm{m}}{\mathrm{s}} .
$$

Based on the above expression, it may be concluded that it is very plausible that protein diffusion induced by bulk shear flows can be observed experimentally, if the shear viscosity (or viscosities) of the bulk fluids can be increased.

Finally, we conclude this paper with our outlook, from the stand point of this work, on study of non-equilibrium dynamics of membrane systems. It is our belief that nonequilibrium behaviour of membrane systems must be investigated, described and understood. It is then our hope that the theory presented in this paper will contribute to that study as a general framework, from which applications to specific concrete problems can grow. Compared to more intuition-based approaches to formulating descriptions of non-equilibrium dynamics of membrane systems, the present formal approach has the virtue that it avoids guesswork and ensures that relevant effects or mechanisms will be included and correctly described in the equations of motion and in the boundary conditions. To be sure, not all of the constitutive relations rendered possible in the general theory are relevant in a specific application to a specific system. But, a systematic analysis of what is relevant or what is not can be made within this framework. If assumptions are made, then the conditions under which the assumptions are valid can also be made clear.

Indeed, we have shown in Sec. 17.2 how the general theory applies to the simple case of non-equilibrium dynamics of single-component membranes and under what limiting conditions the general theory reduces to the earlier descriptions 67. Another obvious case to apply the general theory to is the system of active membranes studied already both experimentally and theoretically [2. That application will be presented in its full detail in a forthcoming paper [35]. In the context of this paper, however, a question naturally arises concerning that application: does the general theory lead to any results that are qualitatively different from the results derived from the theory developed in a more intuitive way in Ref. [2]? The answer, briefly stated, is yes. The general theory differs conceptually from the earlier theory in terms of several new elements: first, the inclusions of the bulk viscous stresses in the driving force for the transverse permeation processes, as stated in Eq. (109), as well as in the driving forces for lateral diffusion processes, as described in Eq. (138); secondly, a new proposal for the conditions of matching between the bulk and the surface hydrodynamic velocities, as formulated in Eq. (110); finally, the prediction of a mechanism where surface-tangential gradients of the bulk pressures can drive lateral diffusions, as discussed in Sec. 7.3. Under the physical conditions that are assumed to hold for the active membranes considered, in particular, the condition that the membranes may be considered impermeable, the first element may be ignored in practice. It is much harder to ignore the other two. In fact, the analysis in Ref. 35] seems to suggest that the last element may contain a plausible theoretical interpretation of certain experimental observations on diffusion dynamics of the active proteins in the membranes.

The general theory may prove to have another potential, which is also demonstrated in its application to the active membranes [35. The potential derives from the underlying philosophy of the theory: that physical effects associated with the microscopic "bulk" regions in contact with the membrane can be reformulated in terms of surface excess quantities, if the microscopic details are not the focus of a description. The potential is fully taken advantage of in the application and renders technically tractable a calculation that would otherwise be prohibitively difficult, if not completely impossible.

The current theory, despite being sufficiently general, is still limited in its scope of coverage of systems or phenomena. We would like to think that its philosophy will lend itself to future development of even more general theories for describing more complex non-equilibrium phenomena.

ML acknowledges the financial support from University of Southern Denmark in the form of a Ph.D. fellowship. PLH and LM would like to thank the Danish National Research Foundation for its financial support in the form of a long-term operating grant awarded to The MEMPHYS-Center for Biomembrane Physics. The authors are grateful to Tove Nyberg for her technical assistance on the figures.

\section{A Transforming density fields}

This short appendix is included to describe a technical point which may sometimes be encountered in a specific calculation based on the theory. In certain situations, it can be convenient to do calculations by using different linear combinations of the physical density fields, $n_{A}$ 's, rather than the fields themselves. An example is that one may want to use, as the "working" density fields, those combinations that have a certain parity when the chosen direction of the normal director to the dividing surface is reversed. ${ }^{8}$

\footnotetext{
8 See for example Ref. [7, where the so-called "sum field" and the "difference field" are used.
} 
A general linear transformation of the $n_{A}$ 's takes the following form,

$$
n_{A}^{\prime}=\sum_{B} n_{B} M_{A}^{B}
$$

where $M_{A}^{B}$ is a constant matrix with $\operatorname{det} M_{A}^{B} \neq 0$. If $n_{A}^{\prime}$ 's are chosen to be the "working" density fields, equations governing the dynamics of the $n^{\prime}{ }_{A}$-fields become the equations to be used in the calculation. In order that the physics of the problem remain invariant under the transformation, other related fields, constants and currents must also be transformed. Specifically, the continuity equations governing the dynamics of $n^{\prime} A^{\prime}$ 's will in fact have the same mathematical forms as those for the $n_{A}$-fields, but, different effective masses, $m^{\prime A}$, s, must be associated with the $n^{\prime}{ }_{A}$-fields, which are given by the transformation

$$
m^{\prime A}=\sum_{B}\left(M^{-1}\right)^{A}{ }_{B} m^{B},
$$

where $\left(M^{-1}\right)_{B}^{A}$ is the inverse of $M^{B}{ }_{A}$, i.e.

$$
\sum_{B} M_{B}^{A}\left(M^{-1}\right)_{C}^{B}=\delta_{C}^{A} .
$$

Moreover, the mass currents associated with the $n^{\prime}{ }_{A}$-fields must also be the transform of the physical currents $j_{A}^{\alpha}$, $j_{A, \mathrm{r}}^{\alpha}, j_{A, \mathrm{~d}}^{\alpha}$ and $\nu_{A, K}$ by the matrix $M^{B}{ }_{A}$, while the associated chemical potentials are the results of the transformation of $\mu^{A}$ by the matrix $\left(M^{-1}\right)^{A}{ }_{B}$.

The transformations of quantities such as $\tilde{\mu}^{A}$ and kinetic constants $\Omega_{A, B}$, where the species indices $A$ and $B$ do not take value $O$, are in general more complicated. If the following conditions,

$$
M_{A}^{O}=0, \quad M_{O}^{A}=0,
$$

are satisfied, however, $\Omega_{A, B}$ still transform according to a relatively simple rule

$$
\Omega_{A B}^{\prime}=\sum_{C, D} \Omega_{C D} M_{A}^{C} M_{B}^{D} .
$$

It may be worth mentioning that the convention which has been used so far of placing the species-label index $A$ as either a superscript or a subscript has been chosen such that it conforms with the general convention of index contraction in matrix algebra.

\section{References}

1. Structure and Dynamics of Membranes, Vol. 1-2, edited by R. Lipowsky and E. Sackmann (Elsevier, Amsterdam, 1995).

2. J.-B. Manneville, P. Bassereau, D. Lévy, and J. Prost, Phys. Rev. Lett. 82, 4356 (1999); J.-B. Manneville, P. Passereau, S. Ramaswamy, and J. Prost, Phys. Rev. E 64, 021908 (2001).

3. W. Helfrich, Z. Naturforsch. 28c, 693 (1973).
4. E. Evans, Biophys. J. 14, 923 (1974).

5. A. Yeung and E. Evans, J. Phys. II 5, 1501 (1995).

6. U. Seifert and S. Langer, Europhys. Lett. 23, 71 (1993).

7. L. Miao, M.A. Lomholt and J. Kleis, Eur. Phys. J. E . 9, 143 (2002).

8. R. Defay, I. Prigogine, and A. Sanfeld, J. Colloid Interface Sci. 58, 498 (1977).

9. D. Bedeaux, A.M. Albano, and P. Mazur, Physica 82A, 438 (1976).

10. A. Onuki, J. Phys. Soc. Jpn. 62, 385 (1993).

11. J. W. Gibbs, The Scientific Papers of J. Willard Gibbs, Vol. 1 : Thermodynamics (Dover Publications Inc., New York, 1961) pp. 33-54.

12. M. Spivak, A comprehensive Introduction to Differential Geometry (Publish or Perish, Boston, Mass., 1970-75).

13. R. Aris, Vectors, Tensors and Basic Equations of Fluid Mechanics (Dover Publications Inc., New York, 1962).

14. R. Defay, I. Prigogine, and A. Bellemans, Surface Tension and Adsorption (Longmans, Green \& Co., London, 1966).

15. S. Ono and S. Kondo, Encyclopedia of Physics, Vol. X: Structure of Liquids (Springer-Verlag, Berlin, 1960), p. 134-280.

16. J. F. Faucon, M. D. Mitov, P. M. Méléard, I. Bivas, and P. Bothorel, J. Phys. 50, 2389 (1989); E. Evans and W. Rawicz, Phys. Rev. Lett. 64, 2094 (1990).

17. S. Paula, A.G. Volkov, A.N. Van Hoek, T.H. Haines, and D.W. Deamer, Biophys. J. 70, 339 (1996).

18. R. D. Kornberg and H. M. McConnell, Biochemistry 10, 1111 (1971); R. Homan and H. J. Pownall, Biochim. Biophys. Acta 938155 (1988).

19. M. A. Lomholt, PhD Thesis, University of Southern Denmark (2004).

20. M. A. Lomholt and L. Miao, cond-mat/0509664

21. P.A. Kralchevsky, J.C. Eriksson and S. Ljunggren, Adv. Coll. and Interface. Sci. 48, 19 (1994).

22. R. Capovilla, J. Guven, J. Phys. A: Math. and Gen. 35, 6233 (2002).

23. E. Evans and R. Skalak, Mechanics and Thermodynamics of Biomembranes (CRC Press., Boca Raton, 1980).

24. I. Prigogine, Introduction to Themodynamics of Irreversible Processes (Thomas., New York, 1955).

25. P.M. Chaikin and T.C. Lubensky, Principles of Condensed Matter Physics (Cambridge University Press, Cambridge, 1995).

26. L.E. Reichl, A Modern Course in Statistical Physics (John Wiley \& Sons Inc., New York, 1998).

27. L. Onsager, Phys. Rev. 37, 405 (1931); ibid. 38, 2265 (1931).

28. H.B.G. Casimir, Rev. Mod. Phys. 17, 343 (1945).

29. H.J. Kreuzer, Nonequilibrium Thermodynamics and its Statistical Foundations (Oxford University Press, Oxford, 1981).

30. W. Cai, T. C. Lubensky, Phys. Rev. E 524251 (1995).

31. J. M. Backer and E. A. Dawudowicz, J. Biol. Chem. 256, 586 (1980); Y. Lange, J. Dolde, and T. L. Steck, ibid. 256, 5321 (1981); P. F. Devaux, Biochemistry 30, 1163 (1991).

32. W. Helfrich, Phys. Rev. Lett. 23, 372 (1969).

33. L.A. Bagatolli, D.D. Binns, D.M. Jameson and J.P. Albanesi, J. Prot. Chem. 21, 383 (2002).

34. M. Weiss, H. Hashimoto, and T. Nilsson, Biophys. J. 84, 4043 (2003).

35. M.A. Lomholt, cond-mat/0510671 M.A. Lomholt, cond-mat/0510703 